\title{
Different Dietary N-3 Polyunsaturated Fatty Acid Formulations Distinctively Modify Tissue Fatty Acid and N-Acylethanolamine Profiles
}

\author{
Elisabetta Murru ${ }^{1,+}$, Paula A. Lopes ${ }^{2,+}\left(\mathbb{D}\right.$, Gianfranca Carta $^{1}\left(\mathbb{D}\right.$, Claudia Manca $^{1}\left(\mathbb{D}\right.$, Armita Abolghasemi ${ }^{1}(\mathbb{D}$,

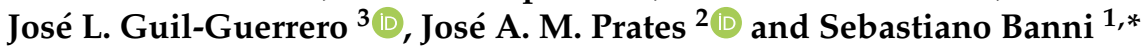 \\ 1 Department of Biomedical Sciences, University of Cagliari, 09042 Monserrato, Cagliari, Italy; \\ m.elisabetta.murru@gmail.com (E.M.); giancarta@unica.it (G.C.); claumanca@hotmail.com (C.M.); \\ armita.abolghasemi@usherbrooke.ca (A.A.) \\ 2 CIISA - Centro de Investigação Interdisciplinar em Sanidade Animal, Faculdade de Medicina Veterinária, \\ Universidade de Lisboa, Avenida da Universidade Técnica, Pólo Universitário do Alto da Ajuda, \\ 1300-477 Lisboa, Portugal; ampalopes@fmv.ulisboa.pt (P.A.L.); japrates@fmv.ulisboa.pt (J.A.M.P.) \\ 3 Departamento de Tecnología de Alimentos, Universidad de Almería, 04120 Almería, Spain; jlguil@ual.es \\ * Correspondence: banni@unica.it \\ + These Authors contributed equally to this work
}

\section{check for} updates

Citation: Murru, E.; Lopes, P.A.; Carta, G.; Manca, C.; Abolghasemi,

A.; Guil-Guerrero, J.L.; Prates, J.A.M.; Banni, S. Different Dietary N-3

Polyunsaturated Fatty Acid

Formulations Distinctively Modify

Tissue Fatty Acid and

N-Acylethanolamine Profiles.

Nutrients 2021, 13, 625 .

https://doi.org/10.3390/nu13020625

Academic Editor: Lindsay Brown

Received: 7 January 2021

Accepted: 9 February 2021

Published: 15 February 2021

Publisher's Note: MDPI stays neutra with regard to jurisdictional claims in published maps and institutional affiliations.

Copyright: (c) 2021 by the authors. Licensee MDPI, Basel, Switzerland. This article is an open access article distributed under the terms and conditions of the Creative Commons Attribution (CC BY) license (https:// creativecommons.org/licenses/by/ $4.0 /)$
Abstract: We investigated the influence of different dietary formulation of n-3 polyunsaturated fatty acids (PUFA) on rat tissue fatty acid (FA) incorporation and consequent modulation of their bioactive metabolite N-acylethanolamines (NAE). For 10 weeks, rats were fed diets with $12 \%$ of fat from milk $+4 \%$ soybean oil and $4 \%$ of oils with different $n-3$ PUFA species: soybean oil as control, linseed oil rich in $\alpha$-linolenic (ALA), Buglossoides arvensis oil rich in ALA and stearidonic acid (SDA), fish oil rich in eicosapentaenoic acid (EPA) and docosahexaenoic acid (DHA), Nannochloropsis microalga oil rich in EPA or Schizochytrium microalga oil rich in DHA. FA and NAE profiles were determined in plasma, liver, brain and adipose tissues. Different dietary n-3 PUFA distinctively influenced tissue FA profiles and consequently NAE tissue concentrations. Interestingly, in visceral adipose tissue the levels of $\mathrm{N}$-arachidonoylethanolamide (AEA) and N-docosahexaenoylethanolamide (DHEA), NAE derived from arachidonic acid (AA) and DHA, respectively, significantly correlated with NAE in plasma, and circulating DHEA levels were also correlated with those in liver and brain. Circulating NAE derived from stearic acid, stearoylethanolamide (SEA), palmitic acid and palmitoylethanolamide (PEA) correlated with their liver concentrations. Our data indicate that dietary n-3 PUFA are not all the same in terms of altering tissue FA and NAE concentrations. In addition, correlation analyses suggest that NAE levels in plasma may reflect their concentration in specific tissues. Given the receptor-mediated tissue specific metabolic role of each NAE, a personalized formulation of dietary n-3 PUFA might potentially produce tailored metabolic effects in different pathophysiological conditions.

Keywords: N-acylethanolamides (NAE); dietary n-3 PUFA; vegetable oils; marine oils; microalga oils

\section{Introduction}

An optimal balance among dietary fatty acids (FA) is widely recommended, in particular the intake of n-6 and n-3 polyunsaturated fatty acids (PUFA) in a 4 to 1 ratio. The therapeutic and preventive benefits of dietary n-3 PUFA have been well documented and most evidence applies to n-3 highly unsaturated fatty acids (HUFA), such as eicosapentaenoic acid (EPA, 20:5n-3) and docosahexaenoic acid (DHA, 22:6n-3), occurring in marine-sourced products [1-3].

Because dietary preferences are resilient and it is difficult to recommend an increase in fish products intake to some consumer groups, a possible solution could be to increase n-3 PUFA intake through vegetable products rich in $\alpha$-linolenic acid (ALA, 18:3n-3), the 
precursor of n-3 HUFA. However, today is evident that dietary n-3 PUFA possess, individually, distinct biological properties. Several studies have already demonstrated that the conversion of ALA into DHA is very limited (10-15\%) because ALA at high concentrations saturates the $\Delta 6$-desaturase enzyme, implicated in the conversion of ALA into stearidonic acid (SDA, 18:4n-3) and 24:5n-3 to 24:6n-3 [4], which are metabolic precursors of DHA in both humans and rodents [5-7]. Therefore, EPA and DHA might be more efficiently incorporated into cell membranes through dietary intake $[1,8]$.

In addition, ALA competes with linoleic acid (LA, 18:2n-6) through a series of desaturation and elongation steps, to form EPA and arachidonic acid (AA, 20:4n-6) respectively, causing a competition between $n-3$ and n-6 biosynthesis [2]. The tissue balance between the two families is therefore critical. In this regard, as an efficient means of increasing EPA concentrations in tissues, it has been suggested that dietary SDA, either from terrestrial plants or from Buglossoides arvensis seeds, was more efficiently converted to EPA than ALA [9-13]. However, other reports did not observe any increase of DHA levels as a result of SDA supplementation [12]. In a previous study, we showed that in rats both ALAand SDA-rich hypercaloric diets increased tissue n-3 HUFA contents leading to a marked improvement on the $n-6 / n-3$ ratio, although lacking effects on DHA biosynthesis [14]. These data were also confirmed in humans supplemented with $4 \mathrm{~g} /$ day of linseed oil for two months, in which the conversion to DHA was only $13 \%$ [15].

Based on the current knowledge on the occurrence of n-3 HUFA in microalgae, much attention has been directed for microalgae lipids as an alternative to fish oil supplements to improve n-3 HUFA levels in tissues [16-18]. However, it remains controversial whether algae n-3 HUFA constitute a better n-3 source than fish oil. In a previous study, we have investigated the influence of n-3 HUFA rich-diets (EPA from Nannochloropsis and DHA from Schizochytrium oils) in comparison with combined EPA- and DHA-rich fish oils on tissues FA profile of rat. We reported that a fish oil diet is more effective in causing tissue incorporation of EPA and DHA than microalgae oils from Nannochloropsis and Schizochytrium, respectively [19].

The physiological properties of n-3 HUFA have been attributed to several mechanisms linked to different metabolic pathways associated with obesity, including insulin resistance, liver and heart steatosis, and hypertension [20,21]. n-3 HUFA-derived NAE, such as Neicosapentaenoylethanolamine (EPEA) from EPA and $\mathrm{N}$-docosahexaenoylethanolamine (DHEA) from DHA have been shown anti-inflammatory and synaptogenic properties [22, 23]. In addition, we also have shown that dietary n-3 HUFA can modify NAE levels in several tissues [24], in animal models [25,26], humans [27,28] and in vitro cell cultures [29]. In fact, dietary EPA and DHA, particularly in the form of phospholipids, by competing for their incorporation with AA, were able to decrease $N$-arachidonoylethanolamine or anandamide (AEA), the AA-derived NAE, which is responsible for some metabolic disorders. However, AEA is involved in different intracellular transduction pathways influencing numerous physiological functions, including neuronal development, neuromodulator processes, energy metabolism, and cardiovascular, respiratory and reproductive functions [30-32].

In rodents, dietary lipids having adequate n-6/n-3 PUFA ratios are currently believed as one means to control appetite and obesity, and to improve skeletal muscle response to glucose and insulin sensitivity by modifying endocannabinoid levels [30-32].

The primary purpose of this study was to investigate whether different dietary n-3 PUFA formulations from vegetable or marine oils can influence n-3 HUFA incorporation and modulate the biosynthesis of NAE in key metabolic tissues of Wistar rats. Moreover, we evaluated whether tissue concentrations of some NAE species are correlated to their precursors and also whether different NAE concentrations in plasma correlate with their own tissue concentrations. 


\section{Materials and Methods}

\subsection{Experimental Diets}

The experimental diets were manufactured at CEIA3-Universidad de Almería (Service of Experimental Diets, http:/ / www.ual.es/stecnicos_spe (accessed on 7 January 2021)). Diets were based on AIN-93M standard formulation for rodents with modified lipid composition (approximately $20 \%$ of total fat, w/w), as follows: milk fat diet (MilkFat), a control with $12 \%$ of fat from milk and $8 \%$ from soybean oil; milk fat diet plus linseed oil (LSO) with $12 \%$ fat from milk, $4 \%$ from soybean oil and $4 \%$ from linseed oil rich in ALA; milk fat diet plus Buglossoides arvensis oil (Buglos) with $12 \%$ fat from milk, $4 \%$ from soybean oil and $4 \%$ from ALA- and SDA-rich Buglos; milk fat diet plus cod liver oil (FO) with $12 \%$ from milk fat, $4 \%$ from soybean oil and $4 \%$ from cod liver oil rich in EPA and DHA; milk fat diet plus Nannochloropsis microalga oil (Nanno) with $12.5 \%$ milk fat, $5.9 \%$ from soybean oil and $2.4 \%$ from EPA-rich Nanno oil; milk fat diet plus Schizochytrium microalga oil (Schy) with $12.2 \%$ from milk fat, $6.5 \%$ from soybean oil and $1.8 \%$ from DHA-rich Schy oil. Diet ingredients were purchased at Dyets inc. (Bethlehem, PA, USA). Buglossoides oil was obtained from Buglossoides arvensis seeds (Ahiflower@ oil, Nature's Crops International, Kensington, Canada) known as the best plant available source of SDA in the nature. Nanno was acquired from Monzón BIOTECH, S.L. (Barcelona, Spain) and Schy was cultivated by Instituto Português do Mar e da Atmosfera (IPMA, Lisboa, Portugal). FA profile of MilkFat, LSO, Buglos, FO, Nanno and Schy diets are shown in Table 1.

Table 1. Fatty acid (FA) profile (\%) in different diets.

\begin{tabular}{ccccccc}
\hline FA & MilkFat & LSO & Buglos & FO & Nanno & Schy \\
\hline $8: 0$ & 2.7 & 1.87 & 3.24 & 1.94 & 2.56 & 3.16 \\
$10: 0$ & 5.99 & 6.37 & 8.06 & 4.42 & 4.44 & 5.02 \\
$12: 0$ & 2.34 & 3.5 & 3.69 & 2.17 & 2.79 & 1.23 \\
$14: 0$ & 8.6 & 7.24 & 8.75 & 9.05 & 8.07 & 8.84 \\
$15: 0$ & $18: 57$ & 0.74 & 0.75 & ND & ND & 2.16 \\
$16: 0$ & 27.43 & 25.29 & 25.00 & 27.82 & 25.94 & 29.12 \\
$16: 1 n-7$ & 0.99 & 0.92 & 0.93 & 2.41 & 4.69 & 1.13 \\
$17: 0$ & ND & 0.38 & ND & 0.37 & 0.34 & 0.66 \\
$18: 0$ & 4.43 & 4.31 & 3.55 & 5.58 & 7.34 & 5.19 \\
$18: 1 n-9$ & 20.39 & 20.87 & 17.7 & 21.82 & 19.35 & 19.43 \\
$18: 1 n-7$ & 0.55 & 0.88 & 0.41 & 0.98 & 0.44 & 0.68 \\
$18: 2 n-6$ & 21.33 & 15.91 & 14.01 & 13.79 & 16.48 & 16.06 \\
$18: 3 n-6$ & ND & ND & 1.54 & ND & ND & ND \\
$18: 3 n-3$ & 2.61 & 10.78 & 7.82 & 1.72 & 1.88 & 1.94 \\
$18: 4 n-3$ & 0.26 & 0.14 & 2.53 & 0.33 & 0.25 & ND \\
$20: 4 n-6$ & ND & ND & ND & ND & 0.75 & ND \\
$20: 5 n-3$ & ND & ND & ND & 1.4 & 4.45 & ND \\
$22: 1 n-11$ & ND & ND & ND & 0.98 & ND & ND \\
$22: 6 n-3$ & ND & ND & ND & 1.49 & ND & 4.08 \\
Others & 1.59 & 0.86 & 2.02 & 1.88 & 0.2 & 1.34 \\
Oil \% & 17.92 & 17.67 & 17.02 & 17.47 & 16.26 & 16.76 \\
SUM & 98.41 & 99.2 & 97.98 & 98.12 & 99.85 & 98.70 \\
\hline ND & & & & & \\
nt & & & & & \\
\end{tabular}

ND, not detectable.

\subsection{Animals and Sample Collection}

Animal facilities and environmental requirements, euthanasia and samples collection were previously described $[14,19]$. Briefly, twenty-four Wistar male rats purchased from Harlan Interfauna Iberica SL (Barcelona, Spain), at the age of 28 days were housed individually and exposed to standard cycles of $12 \mathrm{~h}$ light followed by $12 \mathrm{~h}$ dark at constant temperature $\left(22 \pm 1{ }^{\circ} \mathrm{C}\right)$. After arrival, animals were kept during an adaptation period of 1 week, to end up stress and steady all metabolic conditions. Then, rats were assigned to six body weight-matched groups with four animals each: MilkFat, LSO, Buglos, FO, Nanno and Schy with different dietary n-3 PUFA compositions from vegetable or marine oils. Body weight and feed intake were recorded two times a week. The health status of animals was monitored along the experiment and no casualties were registered. By the end of 10 weeks of experimental trial, rats were fasted for $12 \mathrm{~h}$ and killed by decapitation, under 
light isoflurane (Abbott, IL, USA) anaesthesia. The trunk blood was collected into lithium heparin tubes (Sarstedt, Nümbrecht, Germany) and plasma obtained after centrifugation at $1500 \times g$ for $10 \mathrm{~min}$. Plasma, liver, brain and visceral white adipose tissue (VAT) from retroperitoneal fat depot were removed, weighed and stored at $-80{ }^{\circ} \mathrm{C}$ for measurement of fatty acids, and measurement of AEA endocannabinoid and related compounds (NAE).

\subsection{Lipid Analysis}

\subsubsection{Measurement of Fatty Acids}

Total lipids were extracted from tissues samples (plasma, liver, brain and VAT) according to the method of Folch et al. [33]. Total lipid quantification was performed by the method of Chiang et al. [34]. Aliquots of the lipid fraction were mildly saponified using a procedure in order to obtain unsaturated FA (UFA) for HPLC analysis [35]. All reagents were HPLC grade and purchased from Sigma Chemicals Co. (St. Louis, MO, USA). The separation of FA was carried out using an Agilent 1100 HPLC system (Agilent, Palo Alto, CA, USA) equipped with a diode array detector (DAD). A C-18 Inertsil 5 ODS-2 Chrompack column (Chrompack International BV, Middleburg, The Netherlands) with $5 \mu \mathrm{m}$ particle size and $150 \times 4.6 \mathrm{~mm}$, was used with a mobile phase of $\mathrm{CH}_{3} \mathrm{CN} / \mathrm{H}_{2} \mathrm{O} / \mathrm{CH}_{3} \mathrm{COOH}$ $(70 / 30 / 0.12, v / v / v)$ at a flow rate of $1.5 \mathrm{~mL} / \mathrm{min}$ [36]. Saturated FA (SAFA) were measured as fatty acid methyl esters (FAMEs), by a gas chromatograph (Agilent, Model 6890, Palo Alto) equipped with a flame ionization detector (FID); the split ratio was set at 20:1; the injection port temperature was $270^{\circ} \mathrm{C}$; an autosampler from Agilent (Model 7673, Palo Alto, CA, USA) and a $100 \mathrm{~m}$ HP-88 fused capillary column (Agilent, Palo Alto) were used. Data were acquired by the Agilent ChemStation software system.

\subsubsection{Measurement of N-acylethanolamines (NAE)}

Aliquots of the lipid fraction were used for quantification of NAE compounds. Deuterated NAE and congeners were added as internal standards to the samples before extraction, for quantification by isotope dilution. Internal deuterated standards: $\left[{ }^{2} \mathrm{H}\right]_{8} \mathrm{AEA},\left[{ }^{2} \mathrm{H}\right]_{2} \mathrm{OEA}$, $\left[{ }^{2} \mathrm{H}\right]_{4} \mathrm{PEA},\left[{ }^{2} \mathrm{H}\right]_{3}$ SEA were purchased from Cayman Chemicals (MI, USA). NAE quantification was carried out by an Agilent 1100 HPLC system (Agilent, Palo Alto) equipped with a mass spectrometry (MS) Agilent Technologies QQQ triple quadrupole 6420 with electrospray ionization (ESI) source, using positive mode (ESI+). A C-18 Zorbax Eclipse Plus column (Agilent, Palo Alto) with $5 \mu \mathrm{m}$ particle size and $50 \times 4.6 \mathrm{~mm}$ was used with a mobile phase of $\mathrm{CH}_{3} \mathrm{OH} / \mathrm{H}_{2} \mathrm{O} / \mathrm{CHOOH}(80 / 20 / 0.1, v / v / v)$ at a flow rate of $0.5 \mathrm{~mL} / \mathrm{min}$.

$\mathrm{N}_{2}$ was used as a nebulizing gas with a pressure of $50 \mathrm{psi}$, drying gas at $300{ }^{\circ} \mathrm{C}$ and a flow of $11 \mathrm{~L} / \mathrm{min}$, and $4000 \mathrm{~V}$ capillary voltage. For each standard, the precursor ion $[\mathrm{M}+\mathrm{H}]^{+}$was determined during a full scan (SCAN) in MS and subsequently the obtained product ion (PI) was monitored for each transition in multiple reaction monitoring (MRM) mode in MS/MS. The parameters of source, such as cone voltage or fragmentor (CV) and collision energy (CE) have been optimized for each MRM transition.

Data were acquired by the MassHunter workstation acquisition software (version B.08.02), analyzed with MassHunter software for qualitative (version B.08.00 SP1) and quantitative analyses (version B.09.00). NAE compounds were expressed as mol\% of the sum of total FA measured in the corresponding tissue.

\subsection{Statistical Analysis}

The data are expressed as the mean \pm SEM of moles of each FA and NAE with respect to total FA (mol\%), as specified in the legends.

FA and NAE data were not normally distributed, therefore the differences between the six groups were assessed using nonparametric Kruskal-Wallis test (one-way ANOVA on ranks) followed by Dunn's correction for multiple comparisons. Correlation studies between each NAE and the respective precursor, and between NAE levels in plasma and their concentration in the different tissues, were done using the Spearman correlation coefficient. 
Data were analyzed using GraphPad Prism 6.0 (GraphPad Software Inc., La Jolla, CA, USA) with $p \leq 0.05$ as the cut-off for statistical significance between groups. Data with different superscript letters were significantly different according to the statistical analysis, as specified in the tables, and the statistical significances were indicated: ${ }^{*} p \leq 0.05$; ${ }^{* *} p \leq 0.01 ;{ }^{* * *} p \leq 0.001 ;{ }^{* * *} p \leq 0.0001$, as specified in the figures.

\section{Results}

After 10 weeks of dietary treatment, the parameters associated with rats' growth and amount of food intake did not differ among the five groups containing different concentrations and/or composition of n-3 PUFA compared to MilkFat diet (data not shown).

\subsection{Modification of FA Profiles by n-3 PUFA Diets in Different Tissues}

Tables 2-5 show total FA profiles in several tissues (plasma, liver, brain and VAT) after feeding rats with different dietary n-3 PUFA sources. Tissue FA profiles were strongly influenced by the different dietary FA composition. In fact, in plasma (Table 2) were detected the highest ALA values in LSO- and Buglos-fed animals; EPA in FO- and Nannodiets; DHA reached similar levels in FO- and Schy-fed animals (34\% and 32\%, respectively), despite that DHA intake was 3-times higher in Schy relative to the FO diet. Moreover, DHA, the putative metabolite of ALA, did not increase using LSO or Buglos diets. Furthermore, there was a higher conversion of ALA or ALA+SDA to EPA and docosapentanoic acid (DPA, 22:5n-3) in LSO and Buglos groups, and an elevated incorporation of EPA into tissues and the bioconversion of EPA to DPA in the Nanno group was detected. Simultaneously, a significant decrease in n-6 PUFA levels was observed in FO and Schy groups as compared to MilkFat, and there were significant lower AA levels (about $46 \%$ of the total FA in FO group). The AA biosynthesis did not change in the Schy or Nanno groups, but in Schy LA (the AA precursor) significantly decreased while eicosatrienoic acid (ETA, 20:3n-6), which is the metabolic AA precursor, presented a trend towards increase as compared to MilkFat.

In the liver (Table 3) the FA profile reflected the one found in plasma with a higher incorporation of total n-3 PUFA with all diets and lower total n-6 PUFA levels compared to MilkFat. No significant changes of AA levels where detected when comparing rats fed MilkFat with all groups, however there was a trend of reduction in FO group ( $-30 \%)$ and of increase in Nanno and Schy groups ( $+23 \%$ and $+46 \%$ respectively).

In the brain (Table 4 ) the FA profile slightly changed by using different dietary n-3 PUFA. ALA levels were not different in ALA- and ALA+SDA-enriched LSO and Buglos groups. Moreover, DHA percentage remained unchanged despite its elevated intake using the FO and Schy diets. We found EPA and DPAn-3 increased in all dietary treatments and only EPA significantly in FO group, simultaneously, we found significantly decreased levels of AA and other n-6 PUFA metabolites in the FO-diet group, especially of 22:4n-6 and DPAn-6.

Concerning VAT (Table 5), a significant increase of total n-3 PUFA was detected in LSO group simultaneously to a significant reduction of total n-6 PUFA in LSO and FO groups. In addition, DHA increased significantly in FO and Schy groups.

\subsection{Effect of n-3 PUFA Diets on the Levels of NAE in Different Tissues}

The influence of n-3 PUFA intake with different dietary treatments on the biosynthesis of the main NAE bioactive lipid mediators in relevant tissues of Wistar male rats is detailed in Table 6. Our data indicate that the variations of NAE levels were strongly influenced by tissue FA modifications induced by different dietary n-3 PUFA. A significant positive correlation for nearly all the NAE species with their respective FA precursors in almost all tissues was noted, as described in Figure 1. However, some of them did not follow this trend, such as EPEA in plasma, which had a significant negative correlation with its EPA precursor; also, the LA-derived N-linoleoylethanolamide (LEA) presented a negative, not significant, correlation in plasma, brain and VAT. The oleic acid (OA, 18:1n-9)-derived 
$\mathrm{N}$-oleoylethanolamide (OEA), showed a significant inverse correlation with OA in plasma and VAT.

Table 2. Changes of fatty acid (FA) profile in plasma of Wistar rats fed with different diets.

\begin{tabular}{|c|c|c|c|c|c|c|}
\hline FA & MilkFat & LSO & Buglos & FO & Nanno & Schy \\
\hline ALA, $18: 3 n-3$ & $1.5 \pm 0.3^{\mathrm{a}, \mathrm{b}}$ & $4.8 \pm 0.1^{\mathrm{a}}$ & $3.0 \pm 0.4^{\mathrm{a}}$ & $0.9 \pm 0.0^{\mathrm{a}, \mathrm{b}}$ & $0.9 \pm 0.1^{\mathrm{a}, \mathrm{b}}$ & $0.7 \pm 0.1^{b}$ \\
\hline SDA, $18: 4 n-3$ & $0.1 \pm 0.0^{\mathrm{a}}$ & $0.1 \pm 0.0^{\mathrm{a}}$ & $0.3 \pm 0.0^{\mathrm{a}}$ & $0.1 \pm 0.0^{\mathrm{a}}$ & ND & ND \\
\hline EPA, 20:5n-3 & $0.4 \pm 0.1^{b}$ & $1.8 \pm 0.1^{\mathrm{a}, \mathrm{b}}$ & $1.5 \pm 0.1^{\mathrm{a}, \mathrm{b}}$ & $3.7 \pm 0.1^{\mathrm{a}}$ & $3.9 \pm 0.2^{\mathrm{a}}$ & $0.9 \pm 0.1^{\mathrm{a}, \mathrm{b}}$ \\
\hline DPA, 22:5n-3 & ND & $1.5 \pm 0.2^{\mathrm{a}}$ & $1.0 \pm 0.2^{\mathrm{a}}$ & $1.6 \pm 0.3^{\mathrm{a}}$ & $1.5 \pm 0.2^{\mathrm{a}}$ & ND \\
\hline DHA, 22:6n-3 & $1.0 \pm 0.1^{\mathrm{a}, \mathrm{b}}$ & $1.2 \pm 0.1^{\mathrm{a}, \mathrm{b}}$ & $0.9 \pm 0.1^{\mathrm{a}, \mathrm{b}}$ & $3.6 \pm 0.1^{\mathrm{a}}$ & $0.6 \pm 0.0^{b}$ & $3.4 \pm 0.2^{\mathrm{a}}$ \\
\hline LA, 18:2n-6 & $23.2 \pm 0.8^{a}$ & $21.7 \pm 0.4^{\mathrm{a}, \mathrm{b}}$ & $20.1 \pm 0.5^{\mathrm{a}, \mathrm{b}}$ & $20.6 \pm 0.4^{\mathrm{a}, \mathrm{b}}$ & $21.5 \pm 0.3^{\mathrm{a}, \mathrm{b}}$ & $15.2 \pm 0.6^{b}$ \\
\hline GLA, $18: 3 n-6$ & $0.3 \pm 0.0^{\mathrm{a}, \mathrm{b}}$ & $0.2 \pm 0.0^{\mathrm{a}, \mathrm{b}}$ & $0.7 \pm 0.1^{\mathrm{a}}$ & $0.1 \pm 0.0^{b}$ & $0.2 \pm 0.0^{\mathrm{a}, \mathrm{b}}$ & $0.1 \pm 0.0^{b}$ \\
\hline ETA, $20: 3 n-6$ & $1.1 \pm 0.2^{\mathrm{a}, \mathrm{b}}$ & $0.8 \pm 0.1^{b}$ & $1.9 \pm 0.2^{\mathrm{a}, \mathrm{b}}$ & $0.9 \pm 0.0^{\mathrm{a}, \mathrm{b}}$ & $0.8 \pm 0.1^{\mathrm{a}, \mathrm{b}}$ & $2.5 \pm 0.1^{\mathrm{a}}$ \\
\hline AA, $20: 4 n-6$ & $11.3 \pm 0.7^{\mathrm{a}}$ & $8.4 \pm 0.4^{\mathrm{a}, \mathrm{b}}$ & $8.4 \pm 0.2^{\mathrm{a}, \mathrm{b}}$ & $6.2 \pm 0.3^{b}$ & $12.1 \pm 0.9^{a}$ & $10.6 \pm 0.1^{\mathrm{a}, \mathrm{b}}$ \\
\hline $14: 1 \mathrm{n}-7$ & $0.2 \pm 0.0^{\mathrm{a}}$ & $0.2 \pm 0.0^{\mathrm{a}}$ & $0.3 \pm 0.0^{\mathrm{a}}$ & $0.4 \pm 0.1^{\mathrm{a}}$ & $0.2 \pm 0.0^{\mathrm{a}}$ & $0.3 \pm 0.0^{\mathrm{a}}$ \\
\hline POA, $16: 1 n-7$ & $1.4 \pm 0.2^{\mathrm{a}}$ & $1.6 \pm 0.1^{\mathrm{a}}$ & $1.3 \pm 0.1^{\mathrm{a}}$ & $2.1 \pm 0.2^{\mathrm{a}}$ & $1.8 \pm 0.3^{\mathrm{a}}$ & $1.4 \pm 0.3^{\mathrm{a}}$ \\
\hline OA, $18: 1 n-9$ & $13.8 \pm 1.0^{\mathrm{a}, \mathrm{b}}$ & $15.8 \pm 0.4^{a}$ & $14.7 \pm 0.3^{\mathrm{a}, \mathrm{b}}$ & $15.9 \pm 0.2^{\mathrm{a}}$ & $13.0 \pm 0.7^{\mathrm{a}, \mathrm{b}}$ & $10.9 \pm 0.6^{b}$ \\
\hline MA, 14:0 & $2.1 \pm 0.3^{\mathrm{a}, \mathrm{b}}$ & $0.9 \pm 0.3^{b}$ & $1.4 \pm 0.3^{\mathrm{a}, \mathrm{b}}$ & $1.8 \pm 0.4^{\mathrm{a}, \mathrm{b}}$ & $1.8 \pm 0.1^{\mathrm{a}, \mathrm{b}}$ & $2.8 \pm 0.3^{\mathrm{a}}$ \\
\hline PA, 16:0 & $26.8 \pm 0.2^{a, b}$ & $25.3 \pm 0.4^{\mathrm{b}}$ & $27.1 \pm 0.4^{\mathrm{a}, \mathrm{b}}$ & $28.5 \pm 0.7^{\mathrm{a}, \mathrm{b}}$ & $27.8 \pm 0.3^{\mathrm{a}, \mathrm{b}}$ & $31.2 \pm 0.8^{a}$ \\
\hline SA, 18:0 & $13.4 \pm 0.5^{\mathrm{a}, \mathrm{b}}$ & $13.2 \pm 0.4^{\mathrm{a}, \mathrm{b}}$ & $13.6 \pm 0.9^{\mathrm{a}, \mathrm{b}}$ & $11.1 \pm 0.3^{\mathrm{b}}$ & $11.5 \pm 0.3^{\mathrm{a}, \mathrm{b}}$ & $14.7 \pm 1.0^{\mathrm{a}}$ \\
\hline SAFA & $42.3 \pm 0.5^{\mathrm{a}, \mathrm{b}}$ & $39.4 \pm 0.4^{\mathrm{b}}$ & $42.1 \pm 0.8^{\mathrm{a}, \mathrm{b}}$ & $41.5 \pm 0.7^{\mathrm{a}, \mathrm{b}}$ & $41.1 \pm 0.5^{\mathrm{a}, \mathrm{b}}$ & $48.7 \pm 0.7^{\mathrm{a}}$ \\
\hline MUFA & $15.4 \pm 1.1^{\mathrm{a}, \mathrm{b}}$ & $17.7 \pm 0.5^{\mathrm{a}, \mathrm{b}}$ & $16.3 \pm 0.3^{\mathrm{a}, \mathrm{b}}$ & $18.4 \pm 0.2^{\mathrm{a}}$ & $15.1 \pm 0.9^{\mathrm{a}, \mathrm{b}}$ & $12.7 \pm 0.7^{b}$ \\
\hline PUFA & $39.0 \pm 1.8^{\mathrm{a}, \mathrm{b}}$ & $40.3 \pm 1.1^{\mathrm{a}, \mathrm{b}}$ & $37.7 \pm 1.7^{\mathrm{a}, \mathrm{b}}$ & $37.5 \pm 0.6^{\mathrm{a}, \mathrm{b}}$ & $41.7 \pm 0.7^{\mathrm{a}}$ & $33.8 \pm 1.2^{b}$ \\
\hline PUFAn-3 & $2.9 \pm 0.4^{\mathrm{b}}$ & $9.0 \pm 0.6^{\mathrm{a}}$ & $6.4 \pm 0.8^{\mathrm{a}, \mathrm{b}}$ & $9.5 \pm 0.4^{\mathrm{a}}$ & $6.9 \pm 0.4^{\mathrm{a}, \mathrm{b}}$ & $5.3 \pm 0.4^{\mathrm{a}, \mathrm{b}}$ \\
\hline PUFAn-6 & $35.9 \pm 0.7^{a}$ & $31.0 \pm 0.6^{\mathrm{a}, \mathrm{b}, \mathrm{c}}$ & $31.1 \pm 0.8^{\mathrm{a}, \mathrm{b}}$ & $27.7 \pm 0.5^{\mathrm{b}}$ & $34.6 \pm 1.0^{a, c}$ & $28.4 \pm 0.8^{b, c}$ \\
\hline n-6/n-3 PUFA & $12.8 \pm 1.4^{\mathrm{a}}$ & $3.5 \pm 0.2^{b}$ & $5.1 \pm 0.7^{\mathrm{a}, \mathrm{b}}$ & $2.9 \pm 0.2^{b}$ & $5.0 \pm 0.4^{\mathrm{a}, \mathrm{b}}$ & $5.4 \pm 0.3^{a, b}$ \\
\hline n-3 HUFA score & $0.1 \pm 0.0^{\mathrm{a}}$ & $0.3 \pm 0.0^{\mathrm{a}, \mathrm{b}}$ & $0.2 \pm 0.0^{\mathrm{a}, \mathrm{b}}$ & $0.5 \pm 0.0^{b}$ & $0.3 \pm 0.0^{a, b}$ & $0.3 \pm 0.0^{a, b}$ \\
\hline
\end{tabular}

Data represent mean \pm SEM for four rats per group and are expressed as \% moles/total FA. Values in the same row with different superscript letters are significantly different, $p \leq 0.05$ (one-way ANOVA nonparametric measures with Kruskal-Wallis test). ND, not detectable. ALA, $\alpha$-linolenic acid; SDA, stearidonic acid; EPA, eicosapentaenoic acid; DPA, docosapentaenoic acid; DHA, docosahexaenoic acid; LA, linoleic acid; GLA, $\gamma$-linolenic acid; ETA, eicosatrienoic acid; AA, arachidonic acid; POA, palmitoleic acid; OA, oleic acid; MA, myristic acid; PA, palmitic acid; SA, stearic acid; SAFA, saturated fatty acids; MUFA, monounsaturated fatty acids; PUFA, polyunsaturated fatty acids; HUFA, high unsaturated fatty acids.

Table 3. Changes of fatty acid (FA) profile in liver of Wistar rats fed with different diets.

\begin{tabular}{|c|c|c|c|c|c|c|}
\hline FA & MilkFat & LSO & Buglos & FO & Nanno & Schy \\
\hline ALA, $18: 3 n 3$ & $1.7 \pm 0.1^{\mathrm{a}, \mathrm{b}}$ & $8.6 \pm 0.2^{\mathrm{a}}$ & $5.5 \pm 0.2^{\mathrm{a}}$ & $1.7 \pm 0.0^{\mathrm{a}, \mathrm{b}}$ & $2.0 \pm 0.2^{\mathrm{a}, \mathrm{b}}$ & $1.0 \pm 0.1^{b}$ \\
\hline SDA, $18: 4 n-3$ & $0.2 \pm 0.0^{a, b, c}$ & $0.3 \pm 0.0^{\mathrm{a}, \mathrm{b}}$ & $1.2 \pm 0.1^{\mathrm{a}}$ & $0.2 \pm 0.0^{a, b, c}$ & $0.1 \pm 0.0^{b, c}$ & $0.05 \pm 0.01^{c}$ \\
\hline EPA, 20:5n-3 & $0.5 \pm 0.0^{\mathrm{b}}$ & $1.7 \pm 0.2^{\mathrm{a}, \mathrm{b}}$ & $2.0 \pm 0.0^{a, b}$ & $3.7 \pm 0.1^{\mathrm{a}}$ & $3.7 \pm 0.2^{\mathrm{a}}$ & $1.2 \pm 0.2^{\mathrm{a}, \mathrm{b}}$ \\
\hline DPA, $22: 5 n-3$ & $1.2 \pm 0.3^{b, c}$ & $2.3 \pm 0.1^{\mathrm{a}, \mathrm{b}}$ & $1.9 \pm 0.1^{\mathrm{a}, \mathrm{b}}$ & $2.5 \pm 0.1^{\mathrm{a}, \mathrm{c}}$ & $3.1 \pm 0.1^{\mathrm{a}}$ & $0.8 \pm 0.0^{\mathrm{b}}$ \\
\hline DHA, 22:6n-3 & $1.6 \pm 0.1^{b, c}$ & $2.4 \pm 0.1^{\mathrm{a}, \mathrm{b}, \mathrm{c}}$ & $2.2 \pm 0.1^{\mathrm{a}, \mathrm{b}, \mathrm{c}}$ & $7.4 \pm 0.1^{\mathrm{a}, \mathrm{b}}$ & $1.2 \pm 0.0^{\mathrm{c}}$ & $9.0 \pm 0.1^{\mathrm{a}}$ \\
\hline LA, $18: 2 n-6$ & $29.9 \pm 1.2^{\mathrm{a}}$ & $23.7 \pm 0.3^{\mathrm{a}, \mathrm{b}}$ & $24.7 \pm 0.7^{\mathrm{a}, \mathrm{b}}$ & $19.3 \pm 0.5^{b}$ & $22.3 \pm 0.7^{\mathrm{a}, \mathrm{b}}$ & $20.7 \pm 0.6^{b}$ \\
\hline GLA, $18: 3 n-6$ & $1.0 \pm 0.2^{\mathrm{a}, \mathrm{b}}$ & ND & $2.1 \pm 0.3^{\mathrm{a}}$ & ND & ND & $0.3 \pm 0.0^{\mathrm{b}}$ \\
\hline ETA, 20:3n-6 & $1.3 \pm 0.1^{\mathrm{a}, \mathrm{b}}$ & $1.0 \pm 0.1^{\mathrm{a}, \mathrm{b}}$ & $1.9 \pm 0.2^{\mathrm{a}}$ & $0.7 \pm 0.0^{b}$ & $1.0 \pm 0.1^{\mathrm{a}, \mathrm{b}}$ & $3.1 \pm 0.1^{\mathrm{a}}$ \\
\hline AA, $20: 4 n-6$ & $6.5 \pm 0.1^{\mathrm{a}, \mathrm{b}}$ & $5.0 \pm 0.6^{a, b}$ & $5.1 \pm 0.1^{\mathrm{a}, \mathrm{b}}$ & $4.6 \pm 0.1^{b}$ & $8.0 \pm 0.7^{\mathrm{a}}$ & $9.5 \pm 0.5^{\mathrm{a}}$ \\
\hline DTA, $22: 4 n-6$ & $0.2 \pm 0.0^{\mathrm{a}}$ & $0.1 \pm 0.0^{\mathrm{a}, \mathrm{b}}$ & $0.1 \pm 0.0^{\mathrm{a}, \mathrm{b}}$ & $0.2 \pm 0.1^{\mathrm{a}, \mathrm{b}}$ & $0.1 \pm 0.0^{\mathrm{a}, \mathrm{b}}$ & $0.1 \pm 0.0^{b}$ \\
\hline POA, $16: 1 n 7$ & $3.5 \pm 0.5^{\mathrm{a}}$ & $2.6 \pm 0.1^{\mathrm{a}}$ & $3.0 \pm 0.2^{\mathrm{a}}$ & $4.0 \pm 0.3^{\mathrm{a}}$ & $4.7 \pm 0.9^{\mathrm{a}}$ & $2.7 \pm 0.1^{\mathrm{a}}$ \\
\hline OA, $18: 1 n-9$ & $14.8 \pm 0.6^{\mathrm{a}, \mathrm{b}}$ & $16.2 \pm 0.5^{\mathrm{a}}$ & $14.4 \pm 0.6^{\mathrm{a}, \mathrm{b}}$ & $17.0 \pm 0.6^{\mathrm{a}}$ & $14.6 \pm 1.2^{\mathrm{a}, \mathrm{b}}$ & $10.9 \pm 0.2^{b}$ \\
\hline LAA, 12:0 & $0.1 \pm 0.0^{\mathrm{a}, \mathrm{b}}$ & $0.1 \pm 0.0^{\mathrm{a}, \mathrm{b}}$ & $0.1 \pm 0.0^{\mathrm{a}}$ & $0.1 \pm 0.0^{\mathrm{a}, \mathrm{b}}$ & $0.1 \pm 0.0^{\mathrm{a}, \mathrm{b}}$ & $0.05 \pm 0.0^{\mathrm{b}}$ \\
\hline MA, 14:0 & $1.7 \pm 0.1^{\mathrm{a}}$ & $1.8 \pm 0.0^{\mathrm{a}}$ & $1.9 \pm 0.2^{\mathrm{a}}$ & $2.0 \pm 0.1^{\mathrm{a}}$ & $1.7 \pm 0.2^{\mathrm{a}}$ & $1.0 \pm 0.1^{\mathrm{a}}$ \\
\hline PA, 16:0 & $24.3 \pm 0.2^{\mathrm{a}, \mathrm{b}}$ & $23.2 \pm 0.2^{b}$ & $24.0 \pm 0.5^{\mathrm{a}, \mathrm{b}}$ & $26.8 \pm 0.6^{\mathrm{a}}$ & $24.9 \pm 0.4^{\mathrm{a}, \mathrm{b}}$ & $26.4 \pm 0.5^{\mathrm{a}}$ \\
\hline SA, $18: 0$ & $8.5 \pm 0.9^{\mathrm{a}, \mathrm{b}}$ & $8.0 \pm 0.4^{b}$ & $10.2 \pm 0.8^{\mathrm{a}, \mathrm{b}}$ & $8.6 \pm 0.1^{\mathrm{a}, \mathrm{b}}$ & $10.1 \pm 1.1^{\mathrm{a}, \mathrm{b}}$ & $11.7 \pm 0.4^{\mathrm{a}}$ \\
\hline SAFA & $34.6 \pm 0.9^{\mathrm{a}, \mathrm{b}}$ & $33.5 \pm 0.5^{\mathrm{b}}$ & $36.2 \pm 1.1^{\mathrm{a}, \mathrm{b}}$ & $37.5 \pm 0.8^{\mathrm{a}, \mathrm{b}}$ & $36.9 \pm 1.3^{\mathrm{a}, \mathrm{b}}$ & $39.1 \pm 0.4^{\mathrm{a}}$ \\
\hline MUFA & $18.3 \pm 0.9^{\mathrm{a}, \mathrm{b}}$ & $18.8 \pm 0.5^{\mathrm{a}, \mathrm{b}}$ & $17.4 \pm 0.7^{\mathrm{a}, \mathrm{b}}$ & $21.0 \pm 0.7^{\mathrm{a}}$ & $19.3 \pm 2.1^{\mathrm{a}, \mathrm{b}}$ & $13.7 \pm 0.3^{b}$ \\
\hline PUFA & $44.3 \pm 1.0^{\mathrm{a}, \mathrm{b}}$ & $45.5 \pm 0.9^{\mathrm{a}, \mathrm{b}}$ & $47.2 \pm 0.8^{\mathrm{a}}$ & $40.7 \pm 0.6^{b}$ & $42.0 \pm 1.1^{\mathrm{a}, \mathrm{b}}$ & $45.9 \pm 0.4^{\mathrm{a}, \mathrm{b}}$ \\
\hline PUFAn-3 & $5.1 \pm 0.3^{\mathrm{b}}$ & $15.4 \pm 0.2^{\mathrm{a}}$ & $12.9 \pm 0.2^{\mathrm{a}, \mathrm{b}}$ & $15.5 \pm 0.2^{\mathrm{a}}$ & $10.2 \pm 0.4^{\mathrm{a}, \mathrm{b}}$ & $12.1 \pm 0.2^{\mathrm{a}, \mathrm{b}}$ \\
\hline PUFAn-6 & $38.9 \pm 1.3^{\mathrm{a}}$ & $29.9 \pm 0.7^{\mathrm{a}, \mathrm{b}}$ & $34.0 \pm 0.7^{\mathrm{a}, \mathrm{b}}$ & $24.9 \pm 0.6^{b}$ & $31.4 \pm 1.3^{\mathrm{a}, \mathrm{b}}$ & $33.6 \pm 0.3^{a, b}$ \\
\hline n-6/n-3 PUFA & $7.7 \pm 1.3^{\mathrm{a}}$ & $1.9 \pm 0.0^{\mathrm{b}}$ & $2.6 \pm 0.1^{\mathrm{a}, \mathrm{b}}$ & $1.6 \pm 0.1^{\mathrm{b}}$ & $3.1 \pm 0.5^{\mathrm{a}, \mathrm{b}}$ & $2.8 \pm 0.1^{\mathrm{a}, \mathrm{b}}$ \\
\hline n-3 HUFA score & $0.3 \pm 0.0^{b}$ & $0.5 \pm 0.0^{\mathrm{a}, \mathrm{b}}$ & $0.5 \pm 0.0^{\mathrm{a}, \mathrm{b}}$ & $0.7 \pm 0.0^{\mathrm{a}}$ & $0.5 \pm 0.0^{\mathrm{a}, \mathrm{b}}$ & $0.5 \pm 0.0^{\mathrm{a}, \mathrm{b}}$ \\
\hline
\end{tabular}

Data represent mean \pm SEM for four rats per group and are expressed as \% moles/total FA. Values in the same row with different superscript letters are significantly different, $p \leq 0.05$ (one-way ANOVA nonparametric measures with Kruskal-Wallis test). ND, not detectable. ALA, $\alpha$-linolenic acid; SDA, stearidonic acid; EPA, eicosapentaenoic acid; DPA, docosapentaenoic acid; DHA, docosahexaenoic acid; LA, linoleic acid; GLA, $\gamma$-linolenic acid; ETA, eicosatrienoic acid; AA, arachidonic acid; DTA, docosatetraenoic acid; POA, palmitoleic acid; OA, oleic acid; LAA, lauric acid; MA, myristic acid; PA, palmitic acid; SA, stearic acid; SAFA, saturated fatty acids; MUFA, monounsaturated fatty acids; PUFA, polyunsaturated fatty acids; HUFA, high unsaturated fatty acids. 
Table 4. Changes of fatty acid (FA) profile in brain of Wistar rats fed with different diets.

\begin{tabular}{|c|c|c|c|c|c|c|}
\hline FA & MilkFat & LSO & Buglos & FO & Nanno & Schy \\
\hline ALA, 18:3n 3 & $0.04 \pm 0.01^{\mathrm{a}}$ & $0.05 \pm 0.01^{\mathrm{a}}$ & $0.06 \pm 0.01^{\mathrm{a}}$ & $0.04 \pm 0.01^{\mathrm{a}}$ & $0.04 \pm 0.00^{\mathrm{a}}$ & $0.04 \pm 0.01^{\mathrm{a}}$ \\
\hline EPA, 20:5n-3 & $0.01 \pm 0.00^{\mathrm{b}}$ & $0.04 \pm 0.00^{\mathrm{a}, \mathrm{b}}$ & $0.05 \pm 0.00^{\mathrm{a}, \mathrm{b}}$ & $0.10 \pm 0.01^{\mathrm{a}}$ & $0.05 \pm 0.00^{\mathrm{a}, \mathrm{b}}$ & $0.04 \pm 0.00^{\mathrm{b}}$ \\
\hline DPA, $22: 5 n-3$ & $0.37 \pm 0.07^{\mathrm{a}, \mathrm{b}}$ & $0.50 \pm 0.02^{a, b}$ & $0.53 \pm 0.11^{\mathrm{a}, \mathrm{b}}$ & $0.62 \pm 0.07^{\mathrm{a}, \mathrm{b}}$ & $0.65 \pm 0.05^{\mathrm{a}}$ & $0.27 \pm 0.04^{b}$ \\
\hline DHA, 22:6n-3 & $9.16 \pm 0.26^{a, b}$ & $8.52 \pm 0.14^{b}$ & $9.02 \pm 0.05^{a, b}$ & $9.23 \pm 0.40^{\mathrm{a}, \mathrm{b}}$ & $9.16 \pm 0.25^{a, b}$ & $9.93 \pm 0.33^{\mathrm{a}}$ \\
\hline LA, 18:2n-6 & $1.53 \pm 0.13^{a, b}$ & $1.66 \pm 0.05^{a, b}$ & $1.77 \pm 0.06^{\mathrm{a}, \mathrm{b}}$ & $1.71 \pm 0.05^{\mathrm{a}, \mathrm{b}}$ & $1.88 \pm 0.09^{\mathrm{a}}$ & $1.23 \pm 0.04^{b}$ \\
\hline ETA, 20:3n-6 & $0.36 \pm 0.09^{a, b}$ & $0.39 \pm 0.02^{a, b}$ & $0.62 \pm 0.08^{a}$ & $0.49 \pm 0.01^{\mathrm{a}, \mathrm{b}}$ & $0.31 \pm 0.01^{\mathrm{b}}$ & $0.34 \pm 0.03^{\mathrm{a}, \mathrm{b}}$ \\
\hline AA, $20: 4 n-6$ & $9.55 \pm 0.24^{\mathrm{a}}$ & $8.06 \pm 0.07^{b}$ & $8.99 \pm 0.17^{\mathrm{a}, \mathrm{b}}$ & $7.88 \pm 0.17^{\mathrm{b}}$ & $8.88 \pm 0.06^{\mathrm{a}, \mathrm{b}}$ & $8.43 \pm 0.12^{a, b}$ \\
\hline DTA, $22: 4 n-6$ & $2.07 \pm 0.04^{\mathrm{a}, \mathrm{b}}$ & $1.79 \pm 0.02^{a, b, c}$ & $2.01 \pm 0.03^{a, b}$ & $1.53 \pm 0.02^{\mathrm{c}}$ & $1.88 \pm 0.01^{a, b, c}$ & $1.64 \pm 0.07^{b, c}$ \\
\hline DPA, $22: 5 n-6$ & $0.52 \pm 0.09^{\mathrm{a}}$ & $0.21 \pm 0.03^{\mathrm{a}, \mathrm{b}}$ & $0.24 \pm 0.06^{\mathrm{a}, \mathrm{b}}$ & $0.14 \pm 0.00^{b}$ & $0.27 \pm 0.02^{\mathrm{a}, \mathrm{b}}$ & $0.61 \pm 0.04^{\mathrm{a}}$ \\
\hline POA, $16: 1 n 7$ & $0.78 \pm 0.06^{\mathrm{a}}$ & $0.62 \pm 0.05^{\mathrm{a}}$ & $0.69 \pm 0.04^{\mathrm{a}}$ & $0.83 \pm 0.05^{\mathrm{a}}$ & $0.77 \pm 0.03^{\mathrm{a}}$ & $0.70 \pm 0.02^{\mathrm{a}}$ \\
\hline OA, $18: 1 n-9$ & $19.34 \pm 0.84^{\mathrm{a}}$ & $19.88 \pm 0.39^{a}$ & $20.01 \pm 0.36^{\mathrm{a}}$ & $19.85 \pm 0.81^{\mathrm{a}}$ & $18.88 \pm 0.47^{\mathrm{a}}$ & $20.15 \pm 0.73^{\mathrm{a}}$ \\
\hline LAA, 12:0 & $0.34 \pm 0.03^{\mathrm{a}, \mathrm{b}}$ & $0.22 \pm 0.02^{b}$ & $0.29 \pm 0.02^{a, b}$ & $0.27 \pm 0.03^{a, b}$ & $0.33 \pm 0.02^{a, b}$ & $0.42 \pm 0.04^{\mathrm{a}}$ \\
\hline MA, 14:0 & $2.21 \pm 0.12^{b}$ & $3.06 \pm 0.16^{\mathrm{a}}$ & $2.29 \pm 0.14^{\mathrm{a}, \mathrm{b}}$ & $2.71 \pm 0.16^{a, b}$ & $2.53 \pm 0.08^{a, b}$ & $2.31 \pm 0.13^{a, b}$ \\
\hline PA, 16:0 & $31.35 \pm 0.43^{\mathrm{a}}$ & $31.87 \pm 0.14^{\mathrm{a}}$ & $30.91 \pm 0.38^{a}$ & $31.94 \pm 0.69^{a}$ & $31.73 \pm 0.13^{a}$ & $31.23 \pm 0.27^{\mathrm{a}}$ \\
\hline SA, 18:0 & $19.57 \pm 0.15^{\mathrm{a}}$ & $20.18 \pm 0.31^{\mathrm{a}}$ & $19.89 \pm 0.06^{\mathrm{a}}$ & $20.14 \pm 0.40^{\mathrm{a}}$ & $19.79 \pm 0.12^{\mathrm{a}}$ & $19.64 \pm 0.1^{\mathrm{a}}$ \\
\hline SAFA & $53.46 \pm 0.52^{\mathrm{a}}$ & $55.33 \pm 0.31^{\mathrm{a}}$ & $53.38 \pm 0.46^{\mathrm{a}}$ & $55.06 \pm 1.12^{\mathrm{a}}$ & $54.38 \pm 0.19^{a}$ & $53.60 \pm 0.29^{a}$ \\
\hline MUFA & $20.12 \pm 0.81^{\mathrm{a}}$ & $20.50 \pm 0.37^{\mathrm{a}}$ & $20.70 \pm 0.35^{\mathrm{a}}$ & $20.68 \pm 0.84^{a}$ & $19.65 \pm 0.48^{\mathrm{a}}$ & $20.85 \pm 0.75^{\mathrm{a}}$ \\
\hline PUFA & $24.00 \pm 0.48^{\mathrm{a}}$ & $21.62 \pm 0.17^{b}$ & $23.91 \pm 0.16^{\mathrm{a}, \mathrm{b}}$ & $22.24 \pm 0.68^{a, b}$ & $23.44 \pm 0.48^{\mathrm{a}, \mathrm{b}}$ & $22.88 \pm 0.36^{a, b}$ \\
\hline PUFAn-3 & $9.59 \pm 0.26^{\mathrm{a}}$ & $9.12 \pm 0.14^{\mathrm{a}}$ & $9.65 \pm 0.14^{\mathrm{a}}$ & $9.99 \pm 0.47^{\mathrm{a}}$ & $9.90 \pm 0.30^{\mathrm{a}}$ & $10.28 \pm 0.32^{\mathrm{a}}$ \\
\hline PUFAn-6 & $14.39 \pm 0.28^{\mathrm{a}}$ & $12.49 \pm 0.08^{\mathrm{a}, \mathrm{b}}$ & $14.25 \pm 0.14^{\mathrm{a}}$ & $12.24 \pm 0.22^{b}$ & $13.53 \pm 0.19^{a, b}$ & $12.59 \pm 0.12^{a, b}$ \\
\hline n-6/n-3 PUFA & $1.50 \pm 0.06^{\mathrm{a}}$ & $1.37 \pm 0.04^{\mathrm{a}, \mathrm{b}}$ & $1.48 \pm 0.06^{\mathrm{a}}$ & $1.23 \pm 0.08^{b}$ & $1.37 \pm 0.05^{\mathrm{a}, \mathrm{b}}$ & $1.23 \pm 0.08^{b}$ \\
\hline n-3 HUFA score & $0.43 \pm 0.01^{b}$ & $0.46 \pm 0.01^{\mathrm{a}, \mathrm{b}}$ & $0.45 \pm 0.01^{\mathrm{a}, \mathrm{b}}$ & $0.50 \pm 0.02^{\mathrm{a}}$ & $0.46 \pm 0.01^{\mathrm{a}, \mathrm{b}}$ & $0.48 \pm 0.02^{\mathrm{a}}$ \\
\hline
\end{tabular}

Data represent mean \pm SEM for four rats per group and are expressed as $\%$ moles/total FA. Values in the same row with different superscript letters are significantly different, $p \leq 0.05$ (one-way ANOVA nonparametric measures with Kruskal-Wallis test). ALA, $\alpha$-linolenic acid; EPA, eicosapentaenoic acid; DHA, docosahexaenoic acid; LA, linoleic acid; ETA, eicosatrienoic acid; AA, arachidonic acid; DTA, docosatetraenoic acid; DPA, docosapentaenoic acid; POA, palmitoleic acid; OA, oleic acid; LAA, lauric acid; MA, myristic acid; PA, palmitic acid; SA, stearic acid; SAFA, saturated fatty acids; MUFA, monounsaturated fatty acids; PUFA, polyunsaturated fatty acids; HUFA, high unsaturated fatty acids.

Table 5. Changes of fatty acid (FA) profile in visceral adipose tissue (VAT) of Wistar rats fed with different diets.

\begin{tabular}{|c|c|c|c|c|c|c|}
\hline FA & MilkFat & LSO & Buglos & FO & Nanno & Schy \\
\hline ALA, 18:3n3 & $2.12 \pm 0.06^{\mathrm{a}, \mathrm{b}}$ & $5.88 \pm 0.76^{\mathrm{a}}$ & $5.23 \pm 0.80^{\mathrm{a}}$ & $1.23 \pm 0.12^{b}$ & $1.82 \pm 0.07^{\mathrm{a}, \mathrm{b}}$ & $1.64 \pm 0.06^{\mathrm{a}, \mathrm{b}}$ \\
\hline SDA, $18: 4 n-3$ & $0.01 \pm 0.00^{\mathrm{a}, \mathrm{b}}$ & $0.01 \pm 0.00^{\mathrm{a}, \mathrm{b}}$ & $0.55 \pm 0.14^{\mathrm{a}}$ & $0.09 \pm 0.01^{\mathrm{a}, \mathrm{b}}$ & $0.01 \pm 0.00^{\mathrm{b}}$ & $0.01 \pm 0.00^{\mathrm{a}, \mathrm{b}}$ \\
\hline EPA, 20:5n-3 & $0.02 \pm 0.00^{\mathrm{b}}$ & $0.06 \pm 0.01^{\mathrm{a}, \mathrm{b}}$ & $0.07 \pm 0.01^{\mathrm{a}, \mathrm{b}}$ & $0.19 \pm 0.02^{\mathrm{a}, \mathrm{b}}$ & $0.39 \pm 0.01^{\mathrm{a}}$ & $0.07 \pm 0.01^{\mathrm{a}, \mathrm{b}}$ \\
\hline DPA, 22:5n-3 & $0.06 \pm 0.01^{b}$ & $0.12 \pm 0.02^{\mathrm{a}, \mathrm{b}}$ & $0.06 \pm 0.02^{b}$ & $0.21 \pm 0.02^{\mathrm{a}, \mathrm{b}}$ & $0.34 \pm 0.04^{\mathrm{a}}$ & $0.11 \pm 0.02^{\mathrm{a}, \mathrm{b}}$ \\
\hline DHA, 22:6n-3 & $0.03 \pm 0.00^{b}$ & $0.05 \pm 0.00^{\mathrm{b}}$ & $0.12 \pm 0.01^{\mathrm{a}, \mathrm{b}}$ & $0.40 \pm 0.01^{\mathrm{a}}$ & $0.07 \pm 0.01^{\mathrm{a}, \mathrm{b}}$ & $1.32 \pm 0.08^{a}$ \\
\hline LA, 18:2n-6 & $23.90 \pm 0.17^{\mathrm{a}}$ & $14.81 \pm 1.62^{b}$ & $21.38 \pm 1.49^{\mathrm{a}, \mathrm{b}}$ & $14.76 \pm 0.79^{b}$ & $20.45 \pm 0.83^{a, b}$ & $19.63 \pm 0.78^{a, b}$ \\
\hline GLA, 18:3n-6 & $0.07 \pm 0.01^{\mathrm{a}, \mathrm{b}}$ & $0.08 \pm 0.01^{\mathrm{a}, \mathrm{b}}$ & $0.72 \pm 0.18^{a}$ & $0.05 \pm 0.01^{b}$ & $0.07 \pm 0.00^{\mathrm{a}, \mathrm{b}}$ & $0.07 \pm 0.01^{\mathrm{a}, \mathrm{b}}$ \\
\hline ETA, 20:3n-6 & $0.12 \pm 0.01^{\mathrm{a}, \mathrm{b}}$ & $0.09 \pm 0.02^{b}$ & $0.22 \pm 0.02^{\mathrm{a}}$ & $0.12 \pm 0.01^{\mathrm{a}, \mathrm{b}}$ & $0.15 \pm 0.01^{\mathrm{a}, \mathrm{b}}$ & ND \\
\hline AA, $20: 4 n-6$ & $0.20 \pm 0.02^{\mathrm{a}, \mathrm{b}}$ & $0.14 \pm 0.02^{b}$ & $0.18 \pm 0.01^{\mathrm{a}, \mathrm{b}}$ & $0.15 \pm 0.01^{\mathrm{a}, \mathrm{b}}$ & $0.29 \pm 0.01^{\mathrm{a}}$ & $0.30 \pm 0.02^{a}$ \\
\hline $14: 1 n-5$ & $0.25 \pm 0.02^{\mathrm{a}}$ & $0.23 \pm 0.04^{\mathrm{a}}$ & $0.30 \pm 0.03^{\mathrm{a}}$ & $0.26 \pm 0.02^{\mathrm{a}}$ & $0.36 \pm 0.02^{\mathrm{a}}$ & $0.29 \pm 0.02^{\mathrm{a}}$ \\
\hline POA, 16:1n7 & $1.96 \pm 0.14^{\mathrm{b}}$ & $1.69 \pm 0.27^{\mathrm{b}}$ & $2.52 \pm 0.12^{a, b}$ & $2.91 \pm 0.25^{\mathrm{a}, \mathrm{b}}$ & $3.82 \pm 0.20^{\mathrm{a}}$ & $2.53 \pm 0.41^{\mathrm{a}, \mathrm{b}}$ \\
\hline OA, $18: 1 n-9$ & $27.83 \pm 0.28^{a}$ & $23.69 \pm 3.36^{\mathrm{a}}$ & $32.64 \pm 0.79^{a}$ & $25.47 \pm 1.46^{\mathrm{a}}$ & $26.03 \pm 0.32^{\mathrm{a}}$ & $24.71 \pm 1.60^{\mathrm{a}}$ \\
\hline LAA, 12:0 & $1.70 \pm 0.10^{\mathrm{a}}$ & $1.97 \pm 0.22^{\mathrm{a}}$ & $1.51 \pm 0.05^{\mathrm{a}}$ & $2.06 \pm 0.14^{\mathrm{a}}$ & $1.71 \pm 0.10^{\mathrm{a}}$ & $1.86 \pm 0.15^{\mathrm{a}}$ \\
\hline MA, 14:0 & $5.35 \pm 0.15^{\mathrm{a}, \mathrm{b}}$ & $6.76 \pm 0.75^{\mathrm{a}, \mathrm{b}}$ & $4.83 \pm 0.23^{b}$ & $7.87 \pm 0.46^{\mathrm{a}}$ & $6.03 \pm 0.36^{\mathrm{a}, \mathrm{b}}$ & $6.37 \pm 0.46^{\mathrm{a}, \mathrm{b}}$ \\
\hline PA, 16:0 & $28.16 \pm 0.46^{\mathrm{a}, \mathrm{b}}$ & $34.73 \pm 4.28^{a, b}$ & $23.92 \pm 1.66^{b}$ & $35.65 \pm 1.74^{\mathrm{a}}$ & $28.82 \pm 1.65^{\mathrm{a}, \mathrm{b}}$ & $31.58 \pm 1.80^{a, b}$ \\
\hline SA, 18:0 & $4.66 \pm 0.13^{a}$ & $5.47 \pm 0.47^{\mathrm{a}}$ & $4.00 \pm 0.30^{\mathrm{a}}$ & $5.40 \pm 0.32^{\mathrm{a}}$ & $4.73 \pm 0.49^{a}$ & $4.97 \pm 0.36^{\mathrm{a}}$ \\
\hline SAFA & $30.42 \pm 10.14^{\mathrm{a}, \mathrm{b}}$ & $49.46 \pm 5.68^{a, b}$ & $34.83 \pm 2.17^{b}$ & $51.39 \pm 2.64^{a}$ & $41.68 \pm 2.49^{a, b}$ & $45.52 \pm 2.75^{\mathrm{a}, \mathrm{b}}$ \\
\hline MUFA & $30.04 \pm 0.18^{a, b}$ & $25.60 \pm 3.65^{a, b}$ & $35.46 \pm 0.65^{\mathrm{a}}$ & $28.65 \pm 1.71^{\mathrm{a}, \mathrm{b}}$ & $30.22 \pm 0.44^{\mathrm{a}, \mathrm{b}}$ & $27.53 \pm 1.81^{b}$ \\
\hline PUFA & $26.95 \pm 0.30^{a, b}$ & $21.63 \pm 2.46^{\mathrm{a}, \mathrm{b}}$ & $29.06 \pm 0.80^{\mathrm{a}}$ & $13.20 \pm 4.47^{b}$ & $24.11 \pm 0.88^{\mathrm{a}, \mathrm{b}}$ & $24.37 \pm 0.94^{\mathrm{a}, \mathrm{b}}$ \\
\hline PUFAn-3 & $2.24 \pm 0.07^{b, c}$ & $6.11 \pm 0.77^{a}$ & $6.03 \pm 0.95^{a, b}$ & $2.12 \pm 0.15^{c}$ & $2.61 \pm 0.03^{a, b, c}$ & $3.15 \pm 0.15^{a, b, c}$ \\
\hline PUFAn-6 & $24.33 \pm 0.20^{\mathrm{a}}$ & $15.14 \pm 1.67^{\mathrm{b}}$ & $22.53 \pm 1.33^{a, b}$ & $15.11 \pm 0.81^{\mathrm{b}}$ & $21.01 \pm 0.84^{\mathrm{a}, \mathrm{b}}$ & $20.84 \pm 0.94^{\mathrm{a}, \mathrm{b}}$ \\
\hline n-6/n-3 PUFA & $10.87 \pm 0.54^{\mathrm{a}}$ & $2.51 \pm 0.23^{b}$ & $4.26 \pm 2.30^{\mathrm{a}, \mathrm{b}}$ & $7.16 \pm 0.58^{a, b}$ & $8.03 \pm 0.55^{\mathrm{a}}$ & $6.67 \pm 0.98^{a, b}$ \\
\hline n-3 HUFA score & $0.24 \pm 0.02^{b}$ & $0.48 \pm 0.08^{\mathrm{a}, \mathrm{b}}$ & $0.37 \pm 0.04^{b}$ & $0.73 \pm 0.02^{\mathrm{a}}$ & $0.61 \pm 0.04^{\mathrm{a}, \mathrm{b}}$ & $0.58 \pm 0.11^{\mathrm{a}, \mathrm{b}}$ \\
\hline
\end{tabular}

Data represent mean \pm SEM for four rats per group and are expressed as \% moles/total FA. Values in the same row with different superscript letters are significantly different, $p \leq 0.05$ (one-way ANOVA nonparametric measures with Kruskal-Wallis test). ND, not detectable. ALA, $\alpha$-linolenic acid; SDA, stearidonic acid; EPA, eicosapentaenoic acid; DPA, docosapentaenoic acid; DHA, docosahexaenoic acid; LA, linoleic acid; GLA, $\gamma$-linolenic acid; ETA, eicosatrienoic acid; AA, arachidonic acid; POA, palmitoleic acid; OA, oleic acid; LAA, lauric acid; MA, myristic acid; PA, palmitic acid; SA, stearic acid; SAFA, saturated fatty acids; MUFA, monounsaturated fatty acids; PUFA, polyunsaturated fatty acids; HUFA, high unsaturated fatty acids. 
Table 6. Changes of N-acylethanolamines (NAE) profile in key metabolic tissues of Wistar rats fed with different diets.

\begin{tabular}{|c|c|c|c|c|c|c|}
\hline NAE & MilkFat & LSO & Buglos & FO & Nanno & Schy \\
\hline \multicolumn{7}{|c|}{ Plasma } \\
\hline EPEA & $117.6 \pm 11.2^{\mathrm{a}}$ & $19.7 \pm 3.5^{b}$ & $72.7 \pm 12.3^{a, b}$ & $35.5 \pm 12.4^{\mathrm{a}, \mathrm{b}}$ & $43.5 \pm 10.3^{a, b}$ & $108.0 \pm 7.4^{\mathrm{a}}$ \\
\hline POEA & $11.9 \pm 0.7^{\mathrm{a}}$ & $10.7 \pm 1.2^{\mathrm{a}}$ & $13.7 \pm 2.5^{\mathrm{a}}$ & $14.2 \pm 4.8^{\mathrm{a}}$ & $14.6 \pm 1.7^{\mathrm{a}}$ & $19.2 \pm 0.8^{a}$ \\
\hline LEA & $60.7 \pm 2.9^{a}$ & $52.3 \pm 4.0^{\mathrm{a}}$ & $70.2 \pm 12.2^{a}$ & $44.2 \pm 15.0^{\mathrm{a}}$ & $65.2 \pm 1.9^{a}$ & $67.8 \pm 5.2^{a}$ \\
\hline AEA & $25.1 \pm 1.9^{\mathrm{a}}$ & $17.5 \pm 1.5^{\mathrm{a}}$ & $22.8 \pm 3.0^{\mathrm{a}}$ & $13.1 \pm 4.6^{\mathrm{a}}$ & $22.5 \pm 1.3^{a}$ & $25.9 \pm 1.3^{\mathrm{a}}$ \\
\hline DHEA & $42.3 \pm 3.0^{\mathrm{a}, \mathrm{b}}$ & $37.3 \pm 0.7^{\mathrm{a}, \mathrm{b}}$ & $43.3 \pm 5.1^{\mathrm{a}, \mathrm{b}}$ & $60.0 \pm 2.1^{\mathrm{a}, \mathrm{b}}$ & $37.6 \pm 2.9^{b}$ & $138.8 \pm 7.5^{\mathrm{a}}$ \\
\hline OEA & $142.0 \pm 9.1^{\mathrm{a}}$ & $118.9 \pm 14.7^{\mathrm{a}}$ & $140.0 \pm 14.0^{\mathrm{a}}$ & $118.7 \pm 41.8^{\mathrm{a}}$ & $147.3 \pm 4.3^{\mathrm{a}}$ & $165.6 \pm 10.5^{\mathrm{a}}$ \\
\hline SEA & $237.2 \pm 26.2^{\mathrm{a}, \mathrm{b}}$ & $179.9 \pm 14.6^{\mathrm{a}, \mathrm{b}}$ & $163.9 \pm 16.9^{b}$ & $193.3 \pm 48.8^{\mathrm{a}, \mathrm{b}}$ & $224.5 \pm 10.1^{\mathrm{a}, \mathrm{b}}$ & $319.6 \pm 28.6^{a}$ \\
\hline PEA & \multicolumn{5}{|c|}{ Liver } & $275.2 \pm 6.8^{a}$ \\
\hline EPEA & $52.4 \pm 8.5^{\mathrm{a}, \mathrm{b}}$ & $163.6 \pm 12.9^{a}$ & $27.1 \pm 6.6^{\mathrm{b}}$ & $161.3 \pm 15.3^{\mathrm{a}}$ & $112.2 \pm 15.9^{\mathrm{a}, \mathrm{b}}$ & $52.4 \pm 12.8^{\mathrm{a}, \mathrm{b}}$ \\
\hline POEA & $5.8 \pm 1.2^{\mathrm{a}}$ & $7.9 \pm 1.6^{\mathrm{a}}$ & $4.4 \pm 1.3^{\mathrm{a}}$ & $10.3 \pm 1.4^{\mathrm{a}}$ & $4.9 \pm 0.7^{\mathrm{a}}$ & $4.6 \pm 0.8^{\mathrm{a}}$ \\
\hline LEA & $188.8 \pm 16.7^{\mathrm{a}}$ & $111.4 \pm 7.6^{\mathrm{a}, \mathrm{b}}$ & $105.6 \pm 6.8^{\mathrm{a}, \mathrm{b}}$ & $104.0 \pm 5.1^{\mathrm{a}, \mathrm{b}}$ & $81.3 \pm 8.3^{b}$ & $117.4 \pm 5.2^{\mathrm{a}, \mathrm{b}}$ \\
\hline AEA & $51.1 \pm 9.0^{\mathrm{a}, \mathrm{b}}$ & $34.2 \pm 2.5^{\mathrm{a}, \mathrm{b}}$ & $34.1 \pm 3.9^{\mathrm{a}, \mathrm{b}}$ & $24.3 \pm 4.3^{b}$ & $31.5 \pm 7.1^{\mathrm{a}, \mathrm{b}}$ & $53.8 \pm 3.3^{\mathrm{a}}$ \\
\hline DHEA & $13.2 \pm 1.2^{\mathrm{a}, \mathrm{b}, \mathrm{c}}$ & $13.1 \pm 1.3^{\mathrm{a}, \mathrm{b}, \mathrm{c}}$ & $10.1 \pm 0.5^{b, c}$ & $42.0 \pm 5.3^{\mathrm{a}, \mathrm{b}}$ & $6.3 \pm 1.1^{\mathrm{c}}$ & $83.9 \pm 6.3^{a}$ \\
\hline OEA & $58.2 \pm 13.3^{\mathrm{a}}$ & $56.2 \pm 6.1^{\mathrm{a}}$ & $48.7 \pm 1.3^{\mathrm{a}}$ & $47.0 \pm 4.8^{\mathrm{a}}$ & $32.6 \pm 4.9^{a}$ & $51.2 \pm 6.7^{\mathrm{a}}$ \\
\hline SEA & $32.3 \pm 1.1^{\mathrm{a}, \mathrm{b}}$ & $49.5 \pm 1.7^{\mathrm{a}, \mathrm{b}}$ & $54.8 \pm 5.0^{\mathrm{a}}$ & $50.8 \pm 3.3^{\mathrm{a}, \mathrm{b}}$ & $22.0 \pm 1.8^{b}$ & $60.1 \pm 7.2^{\mathrm{a}}$ \\
\hline PEA & \multicolumn{5}{|c|}{ Brain } & $49.9 \pm 3.0^{\mathrm{a}}$ \\
\hline EPEA & $19.1 \pm 2.9^{b}$ & $30.3 \pm 5.7^{\mathrm{a}, \mathrm{b}}$ & $34.3 \pm 5.6^{\mathrm{a}, \mathrm{b}}$ & $62.0 \pm 6.8^{a}$ & $51.7 \pm 14.8^{\mathrm{a}, \mathrm{b}}$ & $37.2 \pm 6.9^{\mathrm{a}, \mathrm{b}}$ \\
\hline POEA & $110.8 \pm 8.5^{\mathrm{a}}$ & $79.2 \pm 4.9^{\mathrm{a}}$ & $95.8 \pm 5.3^{\mathrm{a}}$ & $110.8 \pm 11.3^{\mathrm{a}}$ & $67.7 \pm 11.0^{\mathrm{a}}$ & $88.4 \pm 7.0^{\mathrm{a}}$ \\
\hline LEA & $313.1 \pm 36.3^{a}$ & $159.6 \pm 3.4^{b}$ & $222.0 \pm 23.0^{\mathrm{a}, \mathrm{b}}$ & $191.2 \pm 19.2^{\mathrm{a}, \mathrm{b}}$ & $148.9 \pm 31.5^{b}$ & $236.6 \pm 26.7^{a, b}$ \\
\hline AEA & $2181.1 \pm 173.0^{\mathrm{a}}$ & $1212.9 \pm 24.8^{b}$ & $1702.2 \pm 69.0^{\mathrm{a}, \mathrm{b}}$ & $1232.6 \pm 62.5^{b}$ & $\underset{\mathrm{a}, \mathrm{b}}{1232.1 \pm 179.1}$ & $1563.1 \pm 88.3^{a, b}$ \\
\hline DHEA & $453.1 \pm 53.2^{\mathrm{a}}$ & $403.6 \pm 3.3^{\mathrm{a}}$ & $363.2 \pm 41.0^{\mathrm{a}}$ & $437.9 \pm 14.0^{\mathrm{a}}$ & $323.3 \pm 39.6^{\mathrm{a}}$ & $468.9 \pm 34.7^{\mathrm{a}}$ \\
\hline OEA & $973.9 \pm 74.0^{\mathrm{a}}$ & $804.9 \pm 21.2^{\mathrm{a}}$ & $809.3 \pm 63.9^{a}$ & $820.4 \pm 50.2^{\mathrm{a}}$ & $619.7 \pm 111.4^{\mathrm{a}}$ & $918.7 \pm 68.8^{a}$ \\
\hline SEA & $321.7 \pm 52.2^{\mathrm{a}}$ & $141.0 \pm 17.9^{\mathrm{a}}$ & $204.7 \pm 81.0^{\mathrm{a}}$ & $251.9 \pm 79.6^{\mathrm{a}}$ & $188.3 \pm 31.2^{\mathrm{a}}$ & $157.0 \pm 11.9^{\mathrm{a}}$ \\
\hline PEA & $684.3 \pm 86.3^{\mathrm{a}}$ & $715.6 \pm 85.5^{\mathrm{a}}$ & $649.0 \pm 67.2^{\mathrm{a}}$ & $684.5 \pm 36.1^{\mathrm{a}}$ & $571.0 \pm 145.4^{\mathrm{a}}$ & $691.7 \pm 65.7^{\mathrm{a}}$ \\
\hline \multicolumn{7}{|c|}{ Visceral adipose tissue (VAT) } \\
\hline POEA & $0.08 \pm 0.00^{\mathrm{a}}$ & $0.09 \pm 0.01^{\mathrm{a}}$ & $0.08 \pm 0.01^{\mathrm{a}}$ & $0.11 \pm 0.01^{\mathrm{a}}$ & $0.11 \pm 0.01^{\mathrm{a}}$ & $0.10 \pm 0.01^{\mathrm{a}}$ \\
\hline LEA & $1.16 \pm 0.10^{\mathrm{a}}$ & $1.02 \pm 0.16^{\mathrm{a}}$ & $0.81 \pm 0.06^{\mathrm{a}}$ & $0.86 \pm 0.09 \mathrm{a}$ & $0.85 \pm 0.05^{\mathrm{a}}$ & $1.01 \pm 0.04^{\mathrm{a}}$ \\
\hline AEA & $0.21 \pm 0.02^{\mathrm{a}, \mathrm{b}}$ & $0.18 \pm 0.02^{\mathrm{a}, \mathrm{b}}$ & $0.15 \pm 0.01^{b}$ & $0.15 \pm 0.02^{b}$ & $0.20 \pm 0.01 \mathrm{a}, \mathrm{b}$ & $0.30 \pm 0.02^{\mathrm{a}}$ \\
\hline DHEA & $0.36 \pm 0.05^{\mathrm{a}, \mathrm{b}}$ & $0.33 \pm 0.04^{\mathrm{a}, \mathrm{b}}$ & $0.23 \pm 0.04^{b}$ & $0.60 \pm 0.02^{\mathrm{a}, \mathrm{b}}$ & $0.22 \pm 0.04^{b}$ & $1.01 \pm 0.06^{\mathrm{a}}$ \\
\hline OEA & $1.48 \pm 0.09^{\mathrm{a}}$ & $1.64 \pm 0.25^{\mathrm{a}}$ & $1.18 \pm 0.04^{\mathrm{a}}$ & $1.40 \pm 0.14^{\mathrm{a}}$ & $1.16 \pm 0.09^{\mathrm{a}}$ & $1.43 \pm 0.08^{\mathrm{a}}$ \\
\hline SEA & $2.63 \pm 0.04^{\mathrm{a}}$ & $3.11 \pm 0.57^{\mathrm{a}}$ & $1.65 \pm 0.17^{\mathrm{a}}$ & $2.35 \pm 0.22^{\mathrm{a}}$ & $2.33 \pm 0.04^{\mathrm{a}}$ & $2.72 \pm 0.23^{a}$ \\
\hline PEA & $1.68 \pm 0.05^{\mathrm{a}}$ & $1.79 \pm 0.28^{\mathrm{a}}$ & $1.38 \pm 0.04^{\mathrm{a}}$ & $1.52 \pm 0.07^{\mathrm{a}}$ & $1.41 \pm 0.11^{\mathrm{a}}$ & $1.85 \pm 0.11^{\mathrm{a}}$ \\
\hline
\end{tabular}

Data represent mean \pm SEM for four rats per group and are expressed as \%moles/total FA, x1000. Values in the same row with different superscript letters are significantly different, $p \leq 0.05$ (one-way ANOVA nonparametric measures with Kruskal-Wallis test). AEA, Narachidonoylethanolamide or anandamide; DHEA, N-docosahexaenoylethanolamide; EPEA, N-eicosapentaenoylethanolamide; LEA, N-linoleoylethanolamide; OEA, N-oleoylethanolamide; PEA, N-palmitoylethanolamine; SEA, N-stearoylethanolamide.

In detail, from data reported in Table 6, the levels of AEA did not change in plasma, liver and VAT, though in the latter tissue, the Schy fed group showed a trend of $42 \%$ increase when compared to MilkFat. However, in the brain from LSO and FO dietary treatments, the AEA biosynthesis was significantly reduced.

The EPEA levels in plasma decreased significantly only with LSO diet, while in the brain increased significantly in the FO group. The DHEA levels were unchanged in all dietary groups with respect to MilkFat diet.

The plasma NAE levels correlation with those in tissues may partly reveal the contribution of each tissue on circulating NAE levels (Figure 2). The plasma levels of EPEA presented a significant negative correlation with liver and, not significantly, with brain and therefore it appeared not to derive from liver or brain tissues, while plasmatic AEA and DHEA levels were significantly positively correlated with all the tissues. Moreover, circulating levels of SEA and PEA were positively correlated with liver levels, and SEA also with brain levels. 


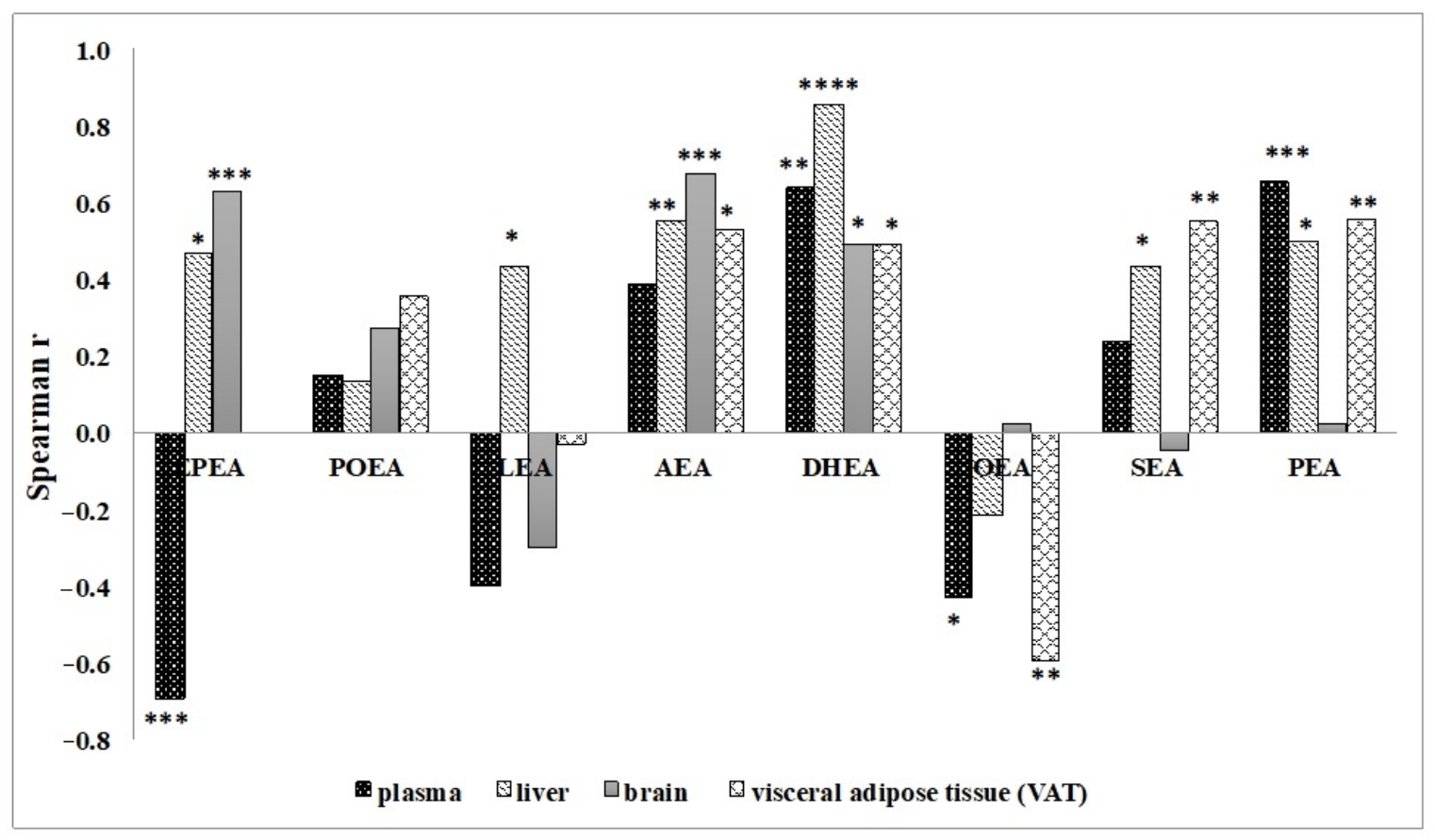

Figure 1. Correlations between NAE species and their respective FA precursors, in plasma, liver, adipose tissue and brain analyzed in Wistar rats fed with different diets. The correlations studies were determined by Spearman correlation coefficient. Statistical significance as follow: ${ }^{*} p \leq 0.05 ;{ }^{* *} p \leq 0.01 ;{ }^{* *} p \leq 0.001 ;{ }^{* * *} p \leq 0.0001$. EPEA, N-eicosapentaenoylethanolamide; POEA, N-pamitoleoylethanolamide; LEA, N-linoleoylethanolamide; AEA, Narachidonoylethanolamide or anandamide; DHEA, N-docosahexaenoylethanolamide; OEA, N-oleoylethanolamide; SEA, N-stearoylethanolamide; PEA, N-palmitoylethanolamine.

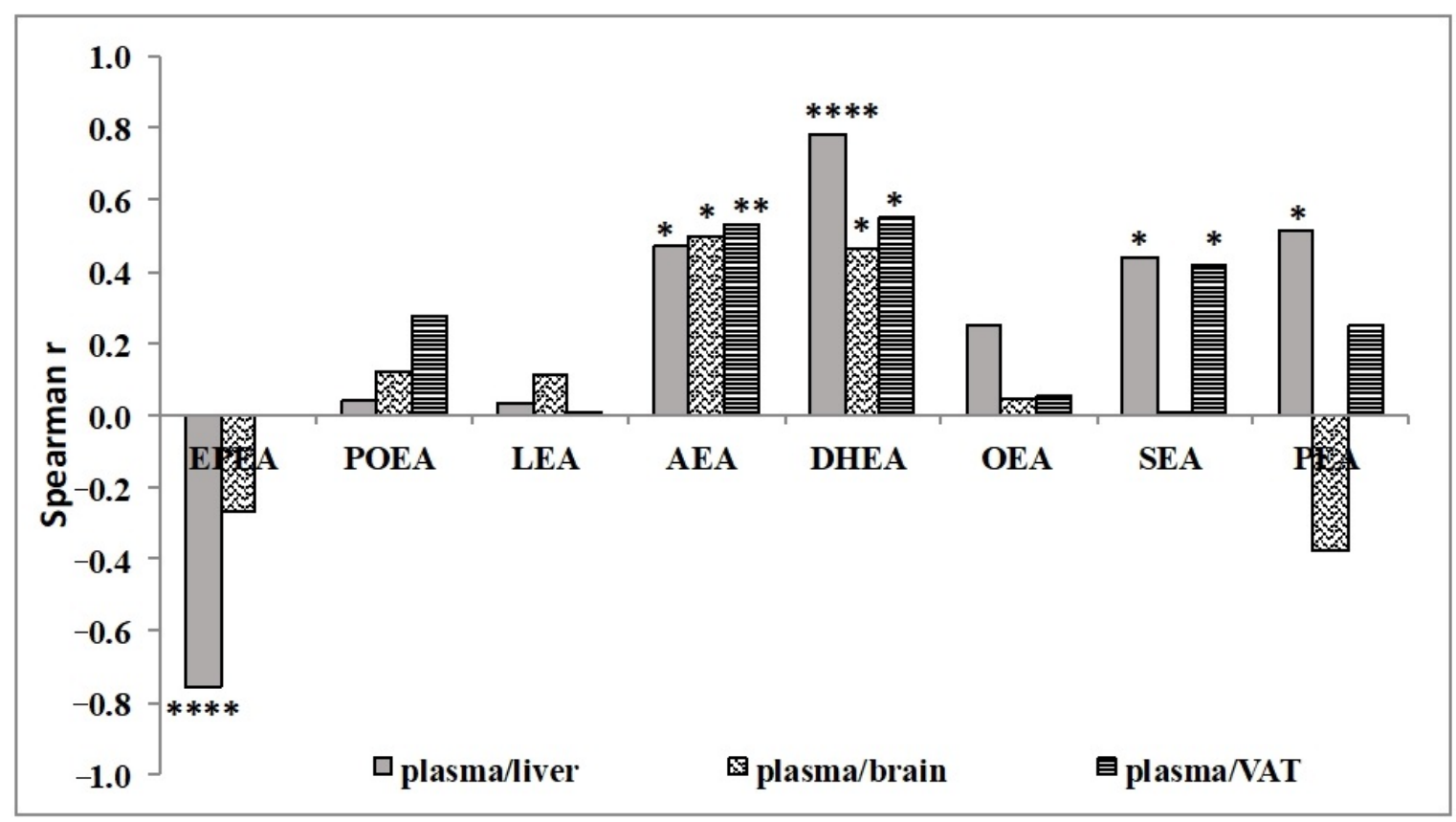

Figure 2. Correlation between levels of NAE species in liver, adipose tissue and brain versus those in plasma in Wistar rats fed with different diets. The correlations studies were determined by Spearman correlation coefficient. Statistical significance as follow: ${ }^{*} p \leq 0.05 ;{ }^{* *} p \leq 0.01 ;{ }^{* * *} p \leq 0.0001$. EPEA, N-eicosapentaenoylethanolamide; POEA, N-pamitoleoylethanolamide; LEA, N-linoleoylethanolamide; AEA, N-arachidonoylethanolamide or anandamide; DHEA, N-docosahexaenoylethanolamide; OEA, N-oleoylethanolamide; SEA, N-stearoylethanolamide; PEA, $\mathrm{N}$-palmitoylethanolamine.; VAT, visceral adipose tissue. 


\section{Discussion}

Our data clearly demonstrate that dietary n-3 PUFA from distinct sources induced differential tissue FA profiles and, consequently, NAE concentrations.

Dietary ALA provided either by LSO or Buglos diets was unable to increase DHA incorporation in any of the tissues analyzed. Interestingly, the inhibition of $\Delta 6$-desaturase by ALA from LSO, but not from Buglos, induced a significant decrease of $16 \%$ AA levels in brain with respect to the control diet, suggesting that a difference of $27 \%$ of dietary ALA may significantly influence AA levels, while the decrease of AA by dietary FO may be attributed to a competition with EPA and DHA for incorporation into phospholipids [8]. These effects on FA modulation appear to be tissue-selective [37-42], and our data confirm that the liver is very sensitive to dietary FA modulation with respect to other tissues [43], especially to brain [44]. Nevertheless, only FO showed a significant increase of n-3 HUFA score in all tissues, underlining the synergistic efficacy of dietary EPA and DHA in modulating tissue fatty acid metabolism with important physiological implications [45].

n-6 and n-3 PUFA, besides being essential components of membrane phospholipids, are precursors of bioactive lipid mediators, such as NAE, with specific receptor-mediated physiological activities. Interestingly, it is now well accepted that dietary modifications of the n-6/n-3 PUFA ratio could affect the biosynthesis of NAE, which may be involved in the modulation of metabolic disorders, as neuroinflammation, pro-inflammatory cytokines release, synaptic plasticity and neurodegenerative conditions [46-48].

As previously demonstrated in studies with rodents and humans $[26,49,50]$, the present data may suggest an enzymatic competition of the biosynthesis of the various NAE species. Indeed, by modifying the relative proportion of n-3 HUFA in the diet and reducing the $n-6 / n-3$ balance of membrane phospholipids, AEA is expected to decrease. In the brain, different dietary n-3 PUFA, as in LSO and FO, significantly reduced AEA concentrations, while in the liver only FO exerted this effect. The reduction of AEA levels might attenuate its effects on the CB1 cannabinoid receptors, the main molecular target of the endogenous partial agonist AEA, which regulate physiological processes in both the central nervous system and peripheral tissues [51-53]. Therefore, in those pathophysiological conditions where is desirable to downregulate an overactive endocannabinoid system, a mixture of EPA and DHA as in FO, and ALA as in LSO, may potentially be a preferred nutritional source of $n-3$ PUFA. In this regard, it would be interesting to evaluate possible positive metabolic outcomes of a dietary mixture of ALA, EPA and DHA.

In this study, we further confirm that tissue concentrations of some NAE species are positively correlated to their precursors. In particular, this is true for NAE species, such as AEA, DHEA, N-palmitoylethanolamine (PEA) and N-stearoylethanolamine (SEA), derived from FA mainly incorporated into phospholipids, since the NAE FA residues derive from those esterified in the sn-1 position of membrane phospholipids [54]. The brain has been found to be less responsive to dietary FA manipulations probably because it is more resistant to changes in the FA profile [44]. On the other hand, in plasma the correlation cannot be ascribed to a direct relationship precursor-product, but most probably by a similar tissue release of the FA precursor and relative NAE. For example, EPEA was negatively correlated to its plasma precursor, contrarily to liver and brain, indicating that only EPA, but not EPEA, is released from tissues into blood.

This study also approached whether different NAE concentrations in plasma correlate with their own tissue concentrations. This issue is relevant in human studies aimed at evaluating tissue modulation of NAE biosynthesis by dietary FA using circulating levels of NAE as biomarkers. Interestingly, our data showed that changes of AEA and DHEA in the VAT significantly correlated with those in the plasma, suggesting that their systemic levels may reflect changes in the adipose tissue [55]. Interestingly, DHEA plasma levels were also correlated to those in liver and brain, while circulating SEA and PEA may significantly reflect their changes only in the liver. Noteworthy, circulating DHEA is the more changeable NAE species across different tissues. However, more specific studies 
using tracers should be carried out to confirm the reliability on the use of circulating NAE as tissue biomarkers.

In conclusion, our data indicate that dietary n-3 PUFA of both vegetable and marine origins differently influence not only FA incorporation and biosynthesis in different tissues, but also selectively affect NAE biosynthesis. In particular, given the receptor-mediated tissue specific metabolic role of each NAE, a personalized formulation of dietary n-3 PUFA might potentially produce tailored metabolic effects in different pathophysiological conditions. In addition, since NAE plasma levels may reflect their concentration in specific tissues, it might be feasible to employ circulating NAE levels for evaluating the nutritional impact of n-3 PUFA in humans.

Author Contributions: E.M., P.A.L. and S.B. conceived the study and participated in its design; E.M. and A.A. carried out the fatty acid and NAE analysis; E.M., G.C. and C.M. performed the statistical analyses; E.M., P.A.L. and S.B. interpreted the data and drafted the manuscript; E.M., P.A.L., G.C., C.M., J.L.G.-G., J.A.M.P. and S.B. contributed to the intellectual content; all authors were involved in critically evaluating the research and revising the final manuscript; all authors have read and approved the final manuscript. All authors have read and agreed to the published version of the manuscript.

Funding: This study was supported by a grant from the University of Cagliari (Fondo Inte-grativo per la Ricerca, FIR 2019) to S.B. and G.C.; by the Spanish Ministry of Science and Innovation through 'Structured lipids: novel dietary strategies for improving human health' grant (AGL/25807/2011) and by Fundação para a Ciência e a Tecnologia (FCT, Lisbon, Portugal) through project UIDB/00276/2020 to CIISA. It is also supported by national funds, through FCT Stimulus of Scientific Employment Program to P.A.L. (DL57/2016/CP1438/CT0007).

Institutional Review Board Statement: All experimental procedures were reviewed by the Ethics Commission of Centro de Investigação Interdisciplinar em Sanidade Animal (CIISA)/Faculdade de Medicina Veterinária (FMV) and approved by the Animal Care Committee of the National Veterinary Authority (Direcção-Geral de Alimentação e Veterinária, Portugal), in accordance with European Union legislation (2010/63/EU Directive).

Informed Consent Statement: Not applicable.

Data Availability Statement: All relevant data are within the manuscript.

Conflicts of Interest: The authors declare no conflict of interest.

\section{Abbreviations}

$\begin{array}{ll}\text { AA } & \text { arachidonic acid } \\ \text { AEA } & \text { N-arachidonoylethanolamide or anandamide } \\ \text { ALA } & \alpha \text {-linolenic acid } \\ \text { Buglos } & \text { Buglossoides arvensis oil } \\ \text { CE } & \text { collision energy } \\ \text { CV } & \text { cone voltage } \\ \text { DAD } & \text { diode array detector } \\ \text { DHA } & \text { docosahexaenoic acid } \\ \text { DHEA } & \text { N-docosahexaenoylethanolamide } \\ \text { DPA } & \text { docosapentanoic acid } \\ \text { DTA } & \text { docosatetraenoic acid } \\ \text { EPA } & \text { eicosapentaenoic acid } \\ \text { EPEA } & \text { N-eicosapentaenoylethanolamide } \\ \text { ESI } & \text { electrospray ionization } \\ \text { ETA } & \text { eicosatrienoic acid } \\ \text { FA } & \text { fatty acids } \\ \text { FAMEs } & \text { fatty acid methyl esters } \\ \text { FID } & \text { flame ionization detector } \\ \text { FO } & \text { fish oil } \\ \text { GLA } & \gamma \text {-linolenic acid }\end{array}$




$\begin{array}{ll}\text { HUFA } & \text { high unsaturated fatty acids } \\ \text { LA } & \text { linoleic acid } \\ \text { LAA } & \text { lauric acid } \\ \text { LEA } & \text { N-linoleoylethanolamide } \\ \text { LSO } & \text { linseed oil } \\ \text { MA } & \text { myristic acid } \\ \text { MilkFat } & \text { milk fat diet } \\ \text { MRM } & \text { multiple reaction monitoring } \\ \text { MS } & \text { mass spectrometry } \\ \text { NAE } & \text { N-acylethanolamides } \\ \text { Nanno } & \text { Nannochloropsis microalga oil } \\ \text { OA } & \text { oleic acid } \\ \text { OEA } & \text { N-oleoylethanolamide } \\ \text { PEA } & \text { N-palmitoylethanolamine } \\ \text { PI } & \text { product ion } \\ \text { PUFA } & \text { polyunsaturated fatty acids } \\ \text { SAFA } & \text { saturated fatty acids } \\ \text { Schy } & \text { Schizochytrium microalga oil } \\ \text { SDA } & \text { stearidonic acid } \\ \text { SA } & \text { stearic acid } \\ \text { SEA } & \text { N-stearoylethanolamide } \\ \text { VAT } & \text { visceral adipose tissue. } \\ & \end{array}$

\section{References}

1. Murru, E.; Banni, S.; Carta, G. Nutritional properties of dietary omega-3-enriched phospholipids. Biomed. Res. Int. 2013, 13, 965417. [CrossRef]

2. Burdge, G.C.; Calder, P.C. Dietary alpha-linolenic acid and health-related outcomes: A metabolic perspective. Nutr. Res. Rev. 2006, 19, 26-52. [CrossRef] [PubMed]

3. Witard, O.C.; Combet, E.; Gray, S.R. Long-chain n-3 fatty acids as an essential link between musculoskeletal and cardio-metabolic health in older adults. Proc. Nutr. Soc. 2020, 79, 47-55. [CrossRef] [PubMed]

4. Voss, A.; Reinhart, M.; Sankarappa, S.; Sprecher, H. The metabolism of 7,10,13,16,19-docosapentaenoic acid to 4,7,10,13,16,19docosahexaenoic acid in rat liver is independent of a 4-desaturase. J. Biol. Chem. 1991, 266, 19995-20000. [CrossRef]

5. Mantzioris, E.; James, M.J.; Gibson, R.A.; Cleland, L.G. Dietary substitution with an alpha-linolenic acid-rich vegetable oil increases eicosapentaenoic acid concentrations in tissues. Am. J. Clin. Nutr. 1994, 59, 1304-1309. [CrossRef] [PubMed]

6. Yamazaki, K.; Fujikawa, M.; Hamazaki, T.; Yano, S.; Shono, T. Comparison of the conversion rates of $\alpha$-linolenic acid (18:3 (n-3)) and stearidonic acid (18:4 (n-3)) to longer polyunsaturated fatty acids in rats. Biochim. Biophys. Acta 1992, 1123, 18. [CrossRef]

7. Huang, Y.S.; Smith, R.S.; Redden, P.R.; Cantrill, R.C.; Horrobin, D.F. Modification of liver fatty acid metabolism in mice by n-3 and n-6 delta 6-desaturase substrates and products. Biochim. Biophys. Acta 1991, 1082, 319-327. [CrossRef]

8. Schuchardt, J.P.; Schneider, I.; Meyer, H.; Neubronner, J.; von Schacky, C.; Hahn, A. Incorporation of EPA and DHA into plasma phospholipids in response to different omega-3 fatty acid formulations-A comparative bioavailability study of fish oil vs. krill oil. Lipids Health Dis. 2011, 10, 145. [CrossRef]

9. Walker, C.G.; Jebb, S.A.; Calder, P.C. Stearidonic acid as a supplemental source of $\omega-3$ polyunsaturated fatty acids to enhance status for improved human health. Nutrition 2013, 29, 363-369. [CrossRef]

10. James, M.J.; Ursin, V.M.; Cleland, L.G. Metabolism of stearidonic acid in human subjects: Comparison with the metabolism of other n-3 fatty acids. Am. J. Clin. Nutr. 2003, 77, 1140-1145. [CrossRef]

11. Krul, E.S.; Lemke, S.L.; Mukherjea, R.; Taylor, M.L.; Goldstein, D.A.; Su, H.; Liu, P.; Lawless, A.; Harris, W.S.; Maki, K.C. Effects of duration of treatment and dosage of eicosapentaenoic acid and stearidonic acid on red blood cell eicosapentaenoic acid content. Prostaglandins Leukot. Essent. Fatty Acids 2012, 86, 51-59. [CrossRef] [PubMed]

12. Harris, W.S.; Lemke, S.L.; Hansen, S.N.; Goldstein, D.A.; DiRienzo, M.A.; Su, H.; Nemeth, M.A.; Taylor, M.L.; Ahmed, G.; George, J. Stearidonic acid-enriched soybean oil increased the omega-3 index, an emerging cardiovascular risk marker. Lipids 2008, 43, 805-811. [CrossRef] [PubMed]

13. Prasad, P.; Anjali, P.; Sreedhar, R.V. Plant-based stearidonic acid as sustainable source of omega-3 fatty acid with functional outcomes on human health. Crit. Rev. Food Sci. Nutr. 2020, 20, 1-13. [CrossRef]

14. Cardoso, C.; Martinho, J.P.; Lopes, P.A.; Martins, S.; Correia, J.; Afonso, C.; Alarcónd, F.J.; González-Fernández, M.J.; Pinto, R.M.; Prates, J.A.; et al. Stearidonic acid combined with alpha-linolenic acid improves lipemic and T neurological markers in a rat model subject to a hypercaloric diet. Prostaglandins Leukot. Essent. Fatty Acids 2018, 135, 137-146. [CrossRef]

15. Murru, E.; Carta, G.; Cordeddu, L.; Melis, M.P.; Desogus, E.; Ansar, H.; Chilliard, Y.; Ferlay, A.; Stanton, C.; Coakley, M.; et al. Dietary Conjugated Linoleic Acid-Enriched Cheeses Influence the Levels of Circulating n-3 Highly Unsaturated Fatty Acids in Humans. Int. J. Mol. Sci. 2018, 19, 1730. [CrossRef] 
16. Martins, D.A.; Custodio, L.; Barreira, L.; Pereira, H.; Ben-Hamadou, R.; Varela, J.; Abu-Salah, K.M. Alternative Sources of n-3 Long-Chain Polyunsaturated Fatty Acids in Marine Microalgae. Mar. Drugs 2013, 11, 2259-2281. [CrossRef]

17. Khozin-Goldberg, I.; Iskandarov, U.; Cohen, Z. LC-PUFA from photosynthetic microalgae: Occurrence, biosynthesis, and prospects in biotechnology. Appl. Microbiol. Biotechnol. 2011, 91, 905-915. [CrossRef]

18. Apt, K.E.; Behrens, P.W. Commercial developments in microalgal biotechnology. J. Phycol. 1999, 35, 215-226. [CrossRef]

19. Lopes, P.A.; Bandarra, N.M.; Martins, S.V.; Martinho, J.; Alfaia, C.M.; Madeira, M.S.; Cardoso, C.; Afonso, C.; Paulo, M.C.; Pinto, R.M.A.; et al. Markers of neuroprotection of combined EPA and DHA provided by fish oil are higher than those of EPA (Nannochloropsis) and DHA (Schizochytrium) from microalgae oils in Wistar rats. Nutr. Metab. 2017, 14, 62. [CrossRef]

20. Carpentier, Y.A.; Portois, L.; Malaisse, W.J. n-3 fatty acids and the metabolic syndrome. Am. J. Clin. Nutr. 2006, 83, 1499s-1504s. [CrossRef] [PubMed]

21. Wang, D.D.; Wu, F.; Zhang, L.Y.; Zhao, Y.C.; Wang, C.C.; Xue, C.H.; Yanagita, T.; Zhang, T.T.; Wang, Y.M. Effects of dietary n-3 PUFA levels in early life on susceptibility to high-fat-diet-induced metabolic syndrome in adult mice. J. Nutr. Biochem. 2020, 89, 108578. [CrossRef]

22. Watson, J.E.; Kim, J.S.; Das, A. Emerging class of omega-3 fatty acid endocannabinoids \& their derivatives. Prostaglandins Other Lipid Mediat. 2019, 143, 106337. [CrossRef]

23. Kim, H.Y.; Spector, A.A. Synaptamide, endocannabinoid-like derivative of docosahexaenoic acid with cannabinoid-independent function. Prostaglandins Leukot. Essent. Fatty Acids 2013, 88, 121-125. [CrossRef]

24. Hansen, H.S.; Artmann, A. Endocannabinoids and nutrition. J. Neuroendocr. 2008, 20, 94-99. [CrossRef] [PubMed]

25. Piscitelli, F.; Carta, G.; Bisogno, T.; Murru, E.; Cordeddu, L.; Berge, K.; Tandy, S.; Cohn, J.S.; Griinari, M.; Banni, S.; et al. Effect of dietary krill oil supplementation on the endocannabinoidome of metabolically relevant tissues from high-fat-fed mice. Nutr. Metab. 2011, 8, 51. [CrossRef] [PubMed]

26. Batetta, B.; Griinari, M.; Carta, G.; Murru, E.; Ligresti, A.; Cordeddu, L.; Giordano, E.; Sanna, F.; Bisogno, T.; Uda, S.; et al. Endocannabinoids May Mediate the Ability of (n-3) Fatty Acids to Reduce Ectopic Fat and Inflammatory Mediators in Obese Zucker Rats. J. Nutr. 2009, 139, 1495-1501. [CrossRef] [PubMed]

27. Banni, S.; Carta, G.; Murru, E.; Cordeddu, L.; Giordano, E.; Sirigu, A.R.; Berge, K.; Vik, H.; Maki, K.C.; Di Marzo, V.; et al. Krill oil significantly decreases 2-arachidonoylglycerol plasma levels in obese subjects. Nutr. Metab. 2011, 8, 1-6. [CrossRef]

28. Berge, K.; Piscitelli, F.; Hoem, N.; Silvestri, C.; Meyer, I.; Banni, S.; Di Marzo, V. Chronic treatment with krill powder reduces plasma triglyceride and anandamide levels in mildly obese men. Lipids Health Dis. 2013, 12, 78. [CrossRef]

29. Matias, I.; Carta, G.; Murru, E.; Petrosino, S.; Banni, S.; Di Marzo, V. Effect of polyunsaturated fatty acids on endocannabinoid and N-acyl-ethanolamine levels in mouse adipocytes. Bba-Mol. Cell Biol. L 2008, 1781, 52-60. [CrossRef]

30. Naughton, S.S.; Mathai, M.L.; Hryciw, D.H.; McAinch, A.J. Fatty Acid Modulation of the Endocannabinoid System and the Effect on Food Intake and Metabolism. Int. J. Endocrinol. 2013. [CrossRef]

31. Kim, J.; Carlson, M.E.; Watkins, B.A. Docosahexaenoyl ethanolamide improves glucose uptake and alters endocannabinoid system gene expression in proliferating and differentiating C2C12 myoblasts. Front. Physiol. 2014, 5, 100. [CrossRef] [PubMed]

32. Bosier, B.; Muccioli, G.G.; Hermans, E.; Lambert, D.M. Functionally selective cannabinoid receptor signalling: Therapeutic implications and opportunities. Biochem. Pharm. 2010, 80, 1-12. [CrossRef] [PubMed]

33. Folch, J.; Lees, M.; Sloane Stanley, G.H. A simple method for the isolation and purification of total lipides from animal tissues. J. Biol. Chem. 1957, 226, 497-509. [CrossRef]

34. Chiang, S.; Gessert, C.; Lowry, O. Colorimetric determination of extracted lipids. An adaptation for microgram amounts of lipids obtained from cerumen. Curr. List Med. Lit. Res. Rep. 1957, 33, 56-113.

35. Banni, S.; Carta, G.; Angioni, E.; Murru, E.; Scanu, P.; Melis, M.P.; Bauman, D.E.; Fischer, S.M.; Ip, C. Distribution of conjugated linoleic acid and metabolites in different lipid fractions in the rat liver. J. Lipid Res. 2001, 42, 1056-1061. [CrossRef]

36. Melis, M.P.; Angioni, E.; Carta, G.; Murru, E.; Scanu, P.; Spada, S.; Banni, S. Characterization of conjugated linoleic acid and its metabolites by RP-HPLC with diode array detector. Eur. J. Lipid Sci. Technol. 2001, 103, 617-621. [CrossRef]

37. Demar, J.J.C.; Ma, K.; Chang, L.; Bell, J.M.; Rapoport, S.I. Alpha-Linolenic acid does not contribute appreciably to docosahexaenoic acid within brain phospholipids of adult rats fed a diet enriched in docosahexaenoic acid. J. Neurochem. 2005, 94, 1063-1076. [CrossRef] [PubMed]

38. Rapoport, S.I.; Rao, J.S.; Igarashi, M. Brain metabolism of nutritionally essential polyunsaturated fatty acids depends on both the diet and the liver. Prostaglandins Leukotr. Essent. Fatty Acids 2007, 77, 251-261. [CrossRef]

39. Igarashi, M.; Ma, K.; Chang, L.; Bell, J.M.; Rapoport, S.I. Rat heart cannot synthesize docosahexaenoic acid from circulating a-linolenic acid be- cause it lacks elongase-2. J. Lipid Res. 2008, 49, 1735-1745. [CrossRef] [PubMed]

40. Igarashi, M.; Ma, K.; Chang, L.; Bell, J.M.; Rapoport, S.I.; DeMar, J.C., Jr. Low liver conversion rate of alpha-linolenic to docosahexaenoic acid in awake rats on a high-docosahexaenoate-containing diet. J. Lipid Res. 2006, 47, 1812-1822. [CrossRef]

41. Wang, Y.; Botolin, D.; Christian, B.; Busik, J.; Xu, J.; Jump, D.B. Tissue-specific, nutritional, and developmental regulation of rat fatty acid elongases. J. Lipid Res. 2005, 46, 706-715. [CrossRef]

42. Igarashi, M.; DeMar, J.C., Jr.; Ma, K.; Chang, L.; Bell, J.M.; Rapoport, S.I. Upregulated liver conversion of alpha-linolenic acid to docosahexaenoic acid in rats on a 15 week n-3 PUFA-deficient diet. J. Lipid Res. 2007, 48, 152-164. [CrossRef] 
43. Bandarra, N.M.; Lopes, P.A.; Martins, S.V.; Ferreira, J.; Alfaia, C.M.; Rolo, E.A.; Correia, J.J.; Pinto, R.M.A.; Ramos-Bueno, R.P.; Batista, I.; et al. Docosahexaenoic acid at the sn-2 position of structured triacylglycerols improved n-3 polyunsaturated fatty acid assimilation in tissues of hamsters. Nutr. Res. 2016, 36, 452-463. [CrossRef]

44. Rodrigues, P.O.; Martins, S.V.; Lopes, P.A.; Ramos, C.; Migueis, S.; Alfaia, C.M.; Pinto, R.M.A.; Rolo, E.A.; Bispo, P.; Batista, I.; et al. Influence of feeding graded levels of canned sardines on the inflammatory markers and tissue fatty acid composition of Wistar rats. Br. J. Nutr. 2014, 112, 309-319. [CrossRef]

45. Stark, K.D. The Percentage of n-3 Highly Unsaturated Fatty Acids in TotalHUFA as a Biomarker for Omega-3 Fatty Acid Status in Tissues. Lipids 2008, 43, 45-53. [CrossRef] [PubMed]

46. Molina-Holgado, E.; Molina-Holgado, F. Mending the broken brain: Neuroimmune interactions in neurogenesis. J. Neurochem. 2010, 114, 1277-1290. [CrossRef] [PubMed]

47. Cassano, T.; Calcagnini, S.; Pace, L.; De Marco, F.; Romano, A.; Gaetani, S. Cannabinoid Receptor 2 Signaling in Neurodegenerative Disorders: From Pathogenesis to a Promising Therapeutic Target. Front. Neurosci. 2017, 11, 30. [CrossRef] [PubMed]

48. Bonnet, A.E.; Marchalant, Y. Potential Therapeutical Contributions of the Endocannabinoid System towards Aging and Alzheimer's Disease. Aging Dis. 2015, 6, 400-405. [CrossRef] [PubMed]

49. Banni, S.; Di Marzo, V. Effect of dietary fat on endocannabinoids and related mediators: Consequences on energy homeostasis, inflammation and mood. Mol. Nutr. Food Res. 2010, 54, 82-92. [CrossRef]

50. Maccarrone, M.; Dainese, E.; Oddi, S. Intracellular trafficking of anandamide: New concepts for signaling. Trends Biochem. Sci. 2010, 35, 601-608. [CrossRef]

51. Brown, I.; Cascio, M.G.; Rotondo, D.; Pertwee, R.G.; Heys, S.D.; Wahle, K.W.J. Cannabinoids and omega-3/6 endocannabinoids as cell death and anticancer modulators. Prog. Lipid Res. 2013, 52, 80-109. [CrossRef]

52. Brown, I.; Cascio, M.G.; Wahle, K.W.J.; Smoum, R.; Mechoulam, R.; Ross, R.A.; Pertwee, R.G.; Heys, S.D. Cannabinoid receptordependent and -independent anti-proliferative effects of omega-3 ethanolamides in androgen receptor-positive and -negative prostate cancer cell lines. Carcinogenesis 2010, 31, 1584-1591. [CrossRef] [PubMed]

53. Pacher, P.; Batkai, S.; Kunos, G. The Endocannabinoid System as an Emerging Target of Pharmacotherapy. Pharmacol. Rev. 2006, 58, 389-462. [CrossRef] [PubMed]

54. Jin, X.H.; Okamoto, Y.; Morishita, J.; Tsuboi, K.; Tonai, T.; Ueda, N. Discovery and characterization of Ca2+-independent phosphatidylethanolarnine $\mathrm{N}$-acyltransferase generating the anandarnide precursor and its congeners. J. Biol. Chem. 2007, 282, 3614-3623. [CrossRef]

55. Fisk, H.L.; Childs, C.E.; Miles, E.A.; Ayres, R.; Noakes, P.S.; Paras-Chavez, C.; Kuda, O.; Kopecky, J.; Antoun, E.; Lillycrop, K.A.; et al. Dysregulation of endocannabinoid concentrations in human subcutaneous adipose tissue in obesity and modulation by omega-3 polyunsaturated fatty acids. Clin. Sci. 2021, 135, 185-200. [CrossRef] 